\title{
Regionalización y relaciones biogeográficas de la Península de Yucatán con base en los patrones de distribución de la familia Leguminosae
}

\author{
The biogeographical regions and relationship of the Yucatán Peninsula based on distribution \\ patterns of the Leguminosae family
}

\author{
Rodrigo Duno-de Stefano ${ }^{\bowtie}$, Lilia Lorena Can-Itza, Agesilas Rivera-Ruiz y Luz María Calvo-Irabién \\ Herbario CICY, Centro de Investigación Científica de Yucatán, A. C. Calle 43 Núm. 130, Col. Chuburná de Hidalgo, 97200 Mérida, Yucatán, México. \\ \roduno@cicy.mx
}

\begin{abstract}
Resumen. Se analizaron los patrones de distribución geográfica de 224 especies de leguminosas nativas de la Península de Yucatán (México) en 48 unidades geográficas operativas ( $0.5^{\circ}$ de latitud y $0.5^{\circ}$ longitud). Las relaciones biogeográficas regionales se determinaron utilizando información de las leguminosas de 12 áreas de América Central, América del Norte, América del Sur y las Antillas. Se emplearon 3 métodos de análisis: coeficiente de Jaccard, análisis de parsimonia (PAE) y un análisis de patrones generales de distribución, como una visualización general de los trazos individuales de Croizat. También se revisaron filogenias de algunos géneros de leguminosas para determinar patrones de evolución geográfica. La mayor riqueza de especies encontrada al sur de la península se asocia con el gradiente climático en sentido norte-sur. Los resultados indican que esta área se puede dividir en 2 áreas biogeográficas (distritos): una franja septentrional y una franja meridional y al menos 5 subdistritos. Los análisis de parsimonia (PAE) y los patrones generales de distribución son congruentes con los análisis de similitud. Todos los resultados sugieren que la Península de Yucatán presenta la mayor afinidad con América Central, y le sigue América del Norte, América del Sur y finalmente las Antillas, mientras que el elemento endémico representa el $6.7 \%$.
\end{abstract}

Palabras clave: biogeografía, flora, Leguminosae, Mesoamérica, análisis de parsimonia de endemismos, Península de Yucatán.

\begin{abstract}
In order to assess biogeographical relationship of the Yucatán Peninsula (Mexico), it was divided in 48 operational geographic units of $0.5^{\circ}$ latitude and longitude and the distribution patterns of 224 native species of the family Leguminosae were analyzed. Biogeographic relations were determined comparing our results with legumes information of 12 neighboring areas of Central, North and South America, and the Caribbean. Three methodologies were used to analyze the information: the Jaccard's similarity coefficient, parsimony analysis of endemism (PAE), and a simple comparison of the general pattern of distribution as a simple model of the individual trace of Croizat. Published phylogenies for some genera were analyzed in order to discuss geographic patterns. The results suggest that the higher species richness found in the southern portion of the Yucatán Peninsula is associated to the north-south climatic gradient present in the peninsula. Based on our results, this area can be divided in 2 biogeographical areas (districts), a northern area with legumes associated to dry habitats and a southern zone with species that grow in more humid climates; 5 subdistrics could also be recognized. The parsimony analysis of endemism and simple comparison of the general pattern of distribution are congruent with the similarity analysis. All the evidence suggests that the Yucatán Peninsula shows more affinity with Central America, followed by North America, South America, and finally the Antilles. On the other hand, the endemic element represents $6.7 \%$ of the legume diversity.
\end{abstract}

Key words: biogeography, flora, Leguminosae, Mesoamerica, parsimony analysis of endemicity, Yucatán Peninsula.

\section{Introducción}

La existencia de la unidad biogeográfica denominada Provincia Biótica Península de Yucatán es bien conocida (Miranda, 1958; Barrera, 1962; Rzedowski, 1978; Estrada-Loera, 1991; Ibarra-Manríquez et al., 2002; Morrone, 2005; Vázquez-Domínguez y Arita, 2010)

Recibido: 04 octubre 2011 ; aceptado: 25 abril 2012 y sus límites han sido evaluados utilizando variables ambientales (clima y fisiografía) y grupos biológicos (plantas, aves, mamíferos, anfibios y reptiles (Lundell, 1934; Goldman y Moore, 1945; Barrera, 1962; Lee, 1980; Ibarra-Manríquez et al., 2002; Espadas Manrique et al., 2003). Un análisis detallado ha llevado a proponer esquemas que reconocen desde 2 hasta 14 subunidades (Ibarra-Manríquez et al., 2002; Morrone, 2005; RamírezBarahona et al., 2009). En la Península de Yucatán, los 
patrones de distribución de organismos se han evaluado desde los siguientes enfoques: 1) patrones de distribución geográfica (Delgadillo, 1984; Chiappy-Jhones, 2001); 2) similitud general (Estrada-Loera, 1991; Ibarra-Manríquez et al., 2002); 3) análisis de parsimonia de endemicidad (Trejo-Torres y Ackerman, 2001, Espadas Manrique et al., 2003); 4) análisis panbiogeográfíco (Morrone, 2001), y 5) una combinación de los 2 últimos (Ramírez-Barahona et al., 2009). En términos de sus afinidades florísticas se ha postulado una relación más cercana con: 1) las Antillas (Standley, 1930; Delgadillo, 1984; ChiappyJhones, 2001; Morrone, 2005, 2006); 2) América Central (Estrada-Loera, 1991; Ibarra-Manríquez et al., 2002), y 3 ) en el caso particular de las dunas costeras, Espejel (1987) sugiere que las 2 regiones contribuyen por igual a su composición florística. Aquí se propone el empleo de la familia Leguminosae como grupo de estudio para evaluar los patrones de regionalización interna y local de la península, admitiendo que la distribución de sus especies es un buen indicador de los patrones florísticos. Leguminosae es la segunda familia de angiospermas más diversa en México con un número estimado entre 1274 y 1724 especies (Sousa y Delgado, 1993; Villaseñor, 2003) y la de mayor diversidad en la Península de Yucatán, con 224 especies nativas que representan aproximadamente el $10 \%$ de la riqueza total de angiospermas (Carnevali et al., 2010). También, en términos de frecuencia y biomasa, es uno de los elementos con mayores valores de importancia ecológica (White y Darwin, 1995; White y Hood, 2004). Los objetivos de este trabajo fueron: 1) utilizar las leguminosas para delimitar subunidades biogeográficas de la Península de Yucatán y compararlas con propuestas previas (Smith, 1941; Barrera, 1962; IbarraManríquez et al., 2002; Ramírez-Barahona et al., 2009); 2) comparar las relaciones florísticas y biogeográficas de la Península de Yucatán con otras áreas de América continental y el Caribe, y 3) evaluar la congruencia del patrón general (límites, distritos y subdistritos) utilizando diferentes tipos de análisis (similitud general, análisis de parsimonia de endemicidad [PAE], patrones generales de distribución y análisis filogenéticos).

\section{Materiales y métodos}

La Península de Yucatán se ubica en la porción SE de la República Mexicana (17 $00^{\circ}-21^{\circ} 45^{\prime}$ N, $86^{\circ} 30^{\prime}-9^{\circ} 30^{\prime}$ $\mathrm{O}$ ); cuenta con una superficie aproximada de $140000 \mathrm{~km}^{2}$, equivalente al $7 \%$ del territorio nacional. Gran parte de su territorio se encuentra limitado por cuerpos de agua: el golfo de México al norte y oeste y el mar Caribe al este; al sur limita con Guatemala, Belice y los estados mexicanos de Tabasco y Chiapas (Fig. 1).
Existe un gradiente climático con menores niveles de precipitación en la porción noroccidental que aumenta hacia la región sureste. En la franja costera noreste predominan los climas Aw (cálido subhúmedos) y en menor proporción los climas BS (semiáridos con 2 subgrupos definidos por el coeficiente de precipitación y temperatura menor o mayor a 22.9) en la costa noroeste. En el caso de la región occidental predominan los climas $\mathrm{Aw}_{0}$, cálido subhúmedos más secos y más cálidos, con menor proporción de lluvias tanto en verano como en invierno y con presencia de sequía intraestival o canícula (Orellana et al., 1999). En la porción poniente se presentan climas $\mathrm{Aw}_{1} \mathrm{o}$ $\mathrm{Aw}_{2}$, más húmedos y menos cálidos, con un mayor porcentaje de precipitación tanto en verano como en invierno y también con presencia de canícula. Únicamente la isla de Cozumel presenta un clima Am (cálido húmedo), con un aporte de lluvias invernales considerable (Orellana et al., 1999). Dicho gradiente climático se debe a la influencia de distintos factores meteorológicos. El promedio de

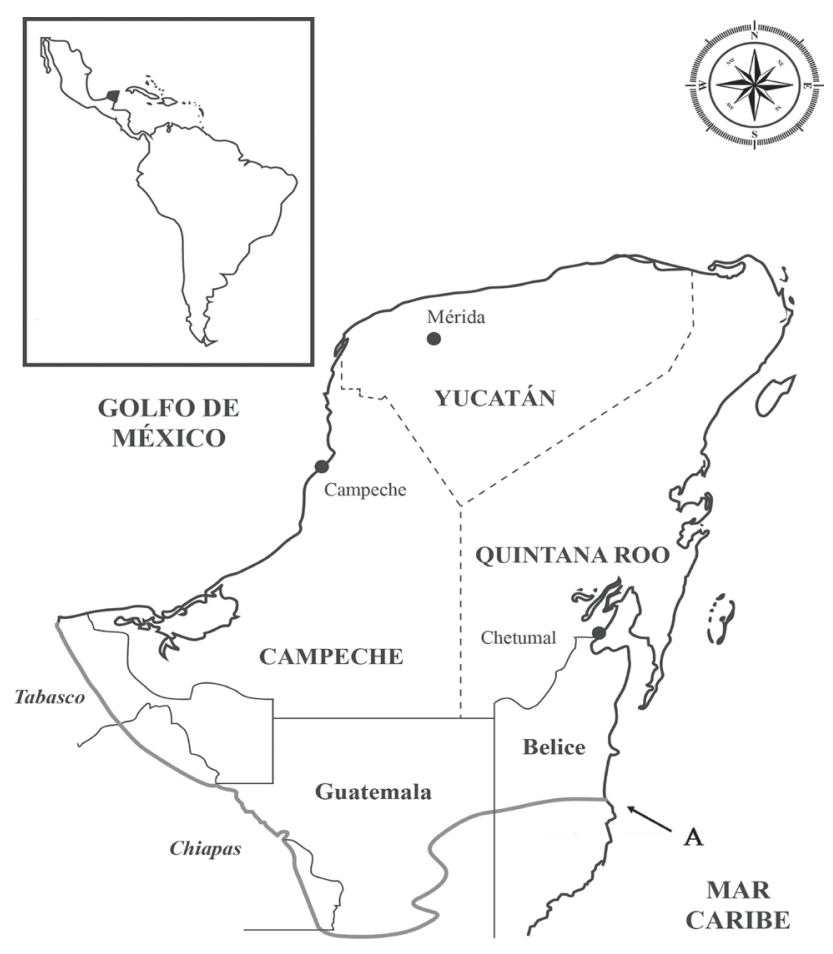

Limites de la Provincia Biótica Peninsula de Yucatán.

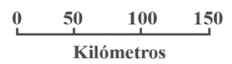

Figura 1. Península de Yucatán, conformada por los estados Campeche, Quintana Roo y Yucatán. La línea A sigue los límites propuestos por Miranda (1958) que incluye parte de los estados de Chiapas y Tabasco (no considerados en el presente trabajo) y también la porción norte de Belice y Guatemala. 
lluvia en verano está dado principalmente por la presencia de los vientos alisios, los cuales se dirigen en dirección noreste-suroeste. La sequía intraestival la determina principalmente la llegada de vaguadas (masas de aire frías) a medio verano. En ciertos años, el aporte de lluvias en verano puede aumentar considerablemente debido a depresiones tropicales y huracanes. La lluvia invernal se genera principalmente por masas de aire polar modificadas o "nortes", con abatimiento térmico en la región y vientos fuertes (Orellana et al., 1999).

La geomorfología del área es de grandes planicies (con alturas menores a $100 \mathrm{~m}$ ), en ocasiones interrumpidas por mesas (planicies elevadas hasta $250 \mathrm{~m}$ ), lomeríos que alcanzan los $350 \mathrm{~m}$ y planicies con lomeríos (Hubp y García, 1999; Vázquez-Domínguez y Arita, 2010). La constitución geológica de la península es en su totalidad de rocas calizas (Ferrusquía-Villafranca, 1993; Hubp y García, 1999; Vázquez-Domínguez y Arita, 2010). La península alberga 5 tipos principales de selvas: selva baja caducifolia, selva mediana subcaducifolia, selva mediana subperennifolia, selva alta perennifolia y la selva baja inundable, ecosistema único en todo México, con 3 asociaciones denominadas tintales, pucteales y mucales (Olmsted et al., 1999). También se reconocen los pastizales inundables y los manglares bajo el nombre genérico de humedales (Olmsted et al., 1999). A diferencia de otras áreas tropicales de México, presenta una baja diversidad florística (161 familias, 956 géneros y 2300 especies) asociada a una relativa homogeneidad climática, topográfica, geomorfológica y edáfica (Carnevali et al., 2010).

Análisis de similitud local y regional. La Península de Yucatán se dividió en cuadros de $0.5^{\circ}$ de latitud por $0.5^{\circ}$ de longitud, con base en los paralelos y meridianos que cruzan su territorio (Fig. 2), de acuerdo con la carta topográfica escala 1:1 000 000. Balleza et al. (2005) sostienen que este tamaño de cuadro es el que mejor resuelve el compromiso entre la información disponible y el número de unidades geográficas operativas (UGO) y son ampliamente utilizados en este tipo de estudios, incluyendo el de la Península de Yucatán (Ibarra-Manríquez et al., 2002). Para la regionalización del estado de Zacatecas, Balleza et al. (2005) procedieron utilizando la familia Asteraceae con el supuesto de que los patrones de distribución de las especies de la familia más abundante son representativos del resto de la flora. Las unidades que incluyen superficies pequeñas con grandes porciones acuáticas se fusionaron con cuadros vecinos a fin de reducir el efecto de diferencia de tamaño y las 63 unidades operativas originales se redujeron a 48. Esta fusión se realizó tomando como criterio la vecindad. Es importante mencionar que en Zacatecas, con una mayor complejidad fisiográfica, la fusión de áreas operativas puede incrementar la riqueza de especies (Balleza et al., 2005), pero no en la Península de Yucatán, donde la homogeneidad fisiográfica es mayor.

La matriz de presencia-ausencia de especies de leguminosas se elaboró utilizando las bases de datos del herbario CICY y de la Red Mexicana de Información sobre Biodiversidad (REMIB, CONABIO). Se realizó una depuración de información con el objetivo de eliminar sinónimos y plantas cultivadas; posteriormente, se construyeron mapas de distribución utilizando la plataforma biótica 4.5 (CONABIO, 2007).

No obstante su enorme tamaño, la base de datos utilizada presenta vacíos de información; por ejemplo, la UGO 33 (que corresponde a la Reserva de la Biosfera de Sian Ka'an) sólo incluía 3 especies. Para minimizar el problema se complementó la matriz (UGO 33) con el listado florístico de Durán y Olmsted (1997). Lo mismo se hizo con las UGO 37 y 38 que corresponden a la Reserva de la Biosfera de Calakmul (Martínez et al., 2001); la UGO 4, para Ría Celestún (Durán et al., 1999); la UGO 12 para la Reserva Ecológica El Edén (Schultz, 2005) y por último, con información de mapas generales de distribución (Flores, 2001). Las especies endémicas se definieron a partir del más reciente listado florístico de la región (Carnevali et al., 2010). No se incluye Prosopis mayana R. Palacios ya que se tiene incertidumbre de su definición con respecto a P. juliflora (Sw.) DC.

Para determinar los patrones de distribución de las especies se utilizó la congruencia entre la distribución geográfica de 2 o más especies mediante técnicas de

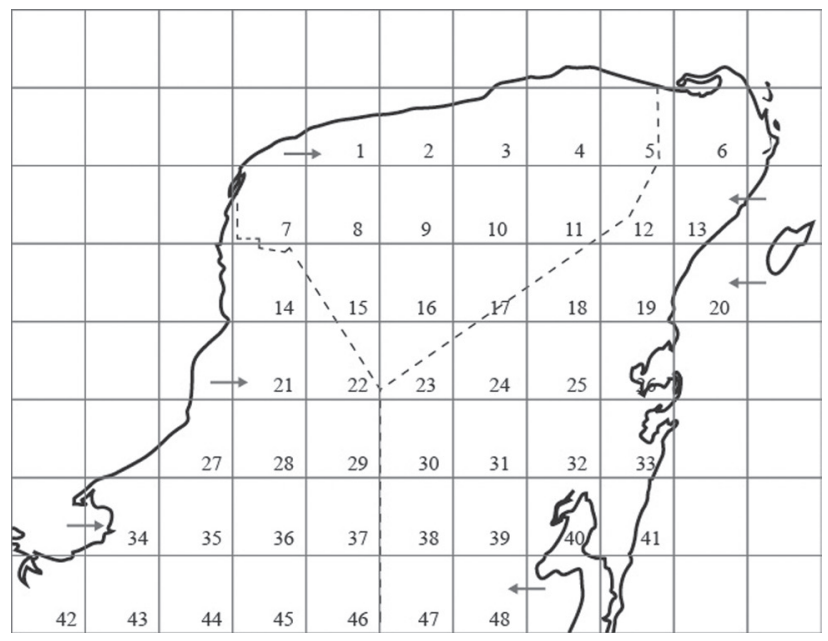

Figura 2. División de la Península de Yucatán en unidades geográficas operativas (UGO). Algunas unidades pequeñas o representadas fundamentalmente por áreas marinas fueron unidas (indicadas con flechas) para homogenizar el área total de todas unidades geográficas operativas. 
clasificación (Murguía y Rojas, 2001), con los métodos numéricos descritos por Jardine (1972), Crovello (1981) y Birks (1987). Se analizó la matriz de presencia-ausencia para 224 especies y 48 UGO; a continuación, la matriz original se transformó en una matriz de similitud, utilizando el coeficiente de similitud de Jaccard (la matriz original puede solicitarse al primer autor). Posteriormente, mediante el método de análisis de grupos pareados sin ponderar, usando la media aritmética (UPGMA), la matriz de similitud se sometió a un análisis de clasificación con el fin de evaluar la similitud florística entre las unidades. Los análisis fueron realizados con el programa Past (Hammer et al., 2001). Con el objetivo de conocer las relaciones fitogeográficas de la península con las áreas vecinas, se fusionaron 7 bandas latitudinales (UGO 1-7, 8-14, etc.) en una nueva matriz que incluyó información de la presencia y ausencia de las leguminosas de los estados de Aguascalientes (Delgado, 1996), Chiapas (Breedlove, 1986), Tabasco (Pérez et al., 2005) y de América Central, América del Sur, América del Norte y Las Antillas (Cuadro 1). Análisis de parsimonia (PAE) local y regional. Se realizó un análisis de parsimonia (PAE), considerándolo como un método particular de evaluación de similitud actual de biotas, bajo la premisa de que sólo la presencia de especies compartidas revela relaciones entre áreas (Trejo-Torres, 2003). No obstante, se reconocen las limitaciones de dicho método en relación con su validez para inferir sobre aspectos de biogeografía histórica (Trejo-Torres, 2003). El PAE requiere matrices de área $v s$. taxones, en las que los estados de carácter son la presencia-ausencia de especies, mientras que las áreas funcionan como taxones, permitiendo así realizar una filogenia de áreas o biotas. Se usaron las mismas matrices anteriores: local (48 UGO y 224 especies) y regional (19 UGO y 998 especies) (Cuadro 1). Las 2 matrices de datos se exportaron a la plataforma Winclada (Nixon, 1999), y con el programa Nona (Goloboff, 1993) se realizó una búsqueda heurística con las siguientes condiciones: multiple TBR+TBR, un máximo de 1000 árboles y 10 repeticiones.

Análisis de distribución y trazos simplificados. Una versión simplificada de los trazos individuales propuestos por Croizat en sus análisis panbiogeográficos (Croizat, 1958), se puede realizar elaborando una lista de las leguminosas de la Península de Yucatán e incluyendo información sobre presencia y ausencia de dichas especies según el siguiente esquema de áreas: Península de Yucatán, América Central, América del Sur, América del Norte, las Antillas, Neotrópico y Pantrópico (Apéndice). Esta información permite cuantificar los patrones generales de distribución para cada leguminosa: patrones ambiguos que corresponden a especies de amplia distribución en el Neotrópico y Pantrópico, y especialmente patrones continentales (de alguna de las siguientes combinaciones de áreas: Península de Yucatán más América Central, América del Sur, América del Norte) y transcaribeños (combinación de alguna área continental y las Antillas).

Análisis de filogenias de grupos selectos. Aunque los métodos panbiogeográficos permiten la descripción de la historia evolutiva de áreas y taxones (Morrone, 2001), otra manera sencilla de inferir aspectos biogeográficos de un género es el uso de los cladogramas producidos en los análisis filogenéticos y sustituir en las ramas terminales

Cuadro 1. Valores de riqueza de especies para regiones seleccionadas del Caribe, Norte América y Centro América utilizadas para el análisis de afinidades regionales

\begin{tabular}{|c|c|c|c|}
\hline País & $\begin{array}{c}\text { Departamento, } \\
\text { estado o provincia }\end{array}$ & $\begin{array}{c}\text { Superficie } \\
\left(\mathrm{km}^{2}\right)\end{array}$ & Referencia \\
\hline Archipiélago de las Bahamas & - & 13940 & Acevedo y Strong, http://botany.si.edu/Antilles/WestIndies/ \\
\hline Belice & $\begin{array}{c}\text { Belice, Corozal y } \\
\text { Orange Walk }\end{array}$ & 22960 & Balick et al., 2000, Tropicos (http://www.tropicos.org/) \\
\hline Estados Unidos de América & $\begin{array}{c}\text { Florida (Dade } \\
\text { County) }\end{array}$ & 2590 & Wunderlin y Hansen, 2008. (http://www.plantatlas.usf.edu/) \\
\hline Guatemala & Petén & 35854 & Standley y Steyermark, 1946, Tropicos (http://www.tropicos.org) \\
\hline Guatemala & Alta Verapaz & 8686 & Standley y Steyermark, 1946, Tropicos (http://www.tropicos.org) \\
\hline Islas Caimán & - & 262 & Acevedo y Strong, http://botany.si.edu/Antilles/WestIndies/ \\
\hline Jamaica & - & 10991 & Acevedo y Strong, http://botany.si.edu/Antilles/WestIndies/ \\
\hline México & Aguascalientes & 73311 & Delgado, 1996 \\
\hline México & Chiapas & 5616 & Breedlove, 1986 \\
\hline México & Tabasco & 22000 & Pérez et al. 2005 \\
\hline Panamá & $\begin{array}{c}\text { Bocas del Toro y } \\
\text { Coclé }\end{array}$ & 9471 & Correa et al., 2004 \\
\hline Puerto Rico & - & 8768 & Acevedo y Strong, http://botany.si.edu/Antilles/WestIndies/ \\
\hline
\end{tabular}


(taxón) del árbol su distribución. Aunque las especies de amplia distribución pueden complicar el análisis de los patrones biogeográficos obtenidos, en especial cuando se trata de especies basales y miembros del grupo externo, los árboles dan una excelente señal de la historia de la ocupación de áreas, incluyendo la posible de su origen. En este sentido, las filogenias disponibles para varios géneros de leguminosas presentes en la Península de Yucatán fueron evaluadas para conocer los patrones geográficos; se acepta que las topologías y la sustitución de taxones terminales por áreas de distribución aportan información sobre el origen y patrón de especiación y ocupación de áreas. Las filogenias evaluadas fueron: Desmanthus Willd. (Luckow 1993, 1997; Luckow y Lavin, 1993), Leucaena Benth. (Hughes, 1998; Hughes et al., 2002), tribu Robinieae y la tribu Sesbanieae (Lavin y Sousa, 1995), tribu Amorphae (Mcmahon y Hufford, 2004), Platymiscium Vogel (Saslis-Lagoudakis et al., 2008) y Swartzia Schreb. (Torke y Schaal, 2008). Como ejemplo, se incluyeron los análisis de 3 géneros: Desmanthus, Platymiscium y Swartzia. Con el programa Mesquite (Maddison y Maddison, 2000) se construyeron los árboles de consenso estricto y se añadió una matriz adicional de especie y áreas de distribución geográfica. Posteriormente, se habilitó la búsqueda de historia de caracteres para visualizar en los nodos ancestrales la ocupación de área.

\section{Resultados}

Análisis de similitud local y regional. Se obtuvieron en total 224 especies de leguminosas nativas de la Península de Yucatán, distribuidas desigualmente en el territorio, que arrojaron diferencias marcadas en la riqueza de especies (Cuadro 2). Las UGO más diversas son 37 y 38, con 156 y 154 especies respectivamente, y las UGO 8 y 9 las

Cuadro 2. Riqueza de especies, variables espaciales y climáticas para las unidades geográficas operativas (UGO) de la Península de Yucatán

\begin{tabular}{|c|c|c|c|c|c|}
\hline$U G O$ & Número de especies & Precipitación promedio anual (PP) & Temperatura promedio anual $(T)$ & $P P / / T$ & Altitud \\
\hline 1 & 82 & 516 & 25.5 & 20.2 & 10 \\
\hline 2 & 79 & 776 & 25.8 & 30.1 & 10 \\
\hline 3 & 80 & 866 & 25.2 & 34.4 & 20 \\
\hline 4 & 84 & 922 & 25.25 & 36.5 & 20 \\
\hline 5 & 93 & 922 & 25 & 36.9 & 20 \\
\hline 6 & 87 & 925 & 25.7 & 36.0 & 20 \\
\hline 7 & 83 & 846 & 26.7 & 31.7 & 10 \\
\hline 8 & 73 & 1112 & 25.7 & 43.3 & 20 \\
\hline 9 & 67 & 1066 & 26 & 41.0 & 20 \\
\hline 10 & 84 & 1166 & 25.15 & 46.3 & 10 \\
\hline 11 & 86 & 1154 & 25.3 & 45.6 & 10 \\
\hline 12 & 90 & 1322 & 25.15 & 52.6 & 40 \\
\hline 13 & 87 & 1204 & 24.8 & 48.5 & 20 \\
\hline 14 & 76 & 1057 & 26.75 & 39.5 & 50 \\
\hline 15 & 88 & 1078 & 24.94 & 43.2 & 140 \\
\hline 16 & 89 & 1078 & 24.92 & 43.3 & 240 \\
\hline 17 & 87 & 1175 & 25.95 & 45.3 & 10 \\
\hline 18 & 82 & 1192 & 25.7 & 46.4 & 40 \\
\hline 19 & 96 & 1192 & 25.3 & 47.1 & 50 \\
\hline 20 & 81 & 1264 & 25.15 & 50.2 & 20 \\
\hline 21 & 109 & 1147 & 26.43 & 43.4 & 240 \\
\hline 22 & 93 & 1120 & 25.4 & 44.1 & 50 \\
\hline 23 & 98 & 987 & 26.1 & 37.8 & 50 \\
\hline 24 & 100 & 1044 & 26.3 & 39.7 & 50 \\
\hline 25 & 112 & 1144 & 26.2 & 43.6 & 40 \\
\hline 26 & 131 & 1266 & 25.55 & 49.5 & 20 \\
\hline 27 & 100 & 1327 & 25.8 & 51.4 & 60 \\
\hline 28 & 92 & 1181 & 24.88 & 47.5 & 140 \\
\hline 29 & 95 & 1114 & 25.76 & 43.2 & 210 \\
\hline 30 & 109 & 1200 & 26.03 & 46.1 & 60 \\
\hline 31 & 101 & 1150 & 25.8 & 44.6 & 140 \\
\hline 32 & 106 & 1348 & 25.8 & 52.2 & 40 \\
\hline 33 & 121 & 1496 & 25.8 & 58.0 & 10 \\
\hline 34 & 103 & 1440 & 25.95 & 55.5 & 20 \\
\hline 35 & 122 & 1383 & 26.05 & 53.1 & 140 \\
\hline 36 & 109 & 1207 & 25.5 & 47.3 & 290 \\
\hline 37 & 154 & 1114 & 25.5 & 43.7 & 150 \\
\hline 38 & 156 & 1200 & 25.4 & 47.2 & 150 \\
\hline 39 & 104 & 1318 & 25.7 & 51.3 & 300 \\
\hline 40 & 112 & 1320 & 25.75 & 51.3 & 50 \\
\hline 41 & 88 & 1448 & 25.8 & 56.1 & 10 \\
\hline
\end{tabular}


Cuadro 2. Continúa

\begin{tabular}{lccccc}
\hline UGO & Número de especies & Precipitación promedio anual $(P P)$ & Temperatura promedio anual $(T)$ & $P P / / T$ & Altitud \\
\hline 42 & 110 & 1750 & 27.15 & 64.5 & 60 \\
43 & 104 & 1287 & 26.7 & 48.2 & 150 \\
44 & 94 & 1513 & 25.86 & 58.5 & 140 \\
45 & 83 & 1300 & 26 & 50.0 & 290 \\
46 & 108 & 1266 & 24.5 & 49.6 \\
47 & 120 & 1266 & 25.8 & 51.7 \\
48 & 121 & 1352 & 100 & 52.4 \\
\hline
\end{tabular}

menos diversas, con 77 y 67 especies, respectivamente. Al menos 20 especies presentan una distribución amplia (más del 90\% de las UGO): Acaciella angustissima, Aeschynomene americana, Acacia gaumeri, A. pennatula, Bauhinia divaricata, Caesalpinia yucatanense, Dalbergia glabra, Desmanthus virgatus, Desmodium glabrum, Desmodium affine, Enterolobium cyclocarpum, Haematoxylum campechianum, Leucaena leucocephala, Lysiloma latisiliquum, Mimosa bahamensis, Piscidia piscipula, Rhynchosia minima, Senna atomaria, Senna racemosa y Zapoteca formosa. Por otro lado, 10 especies están presentes exclusivamente en una UGO: Calliandra caeciliae (UGO 33), Chamaecrista chamaecristoides (UGO 34), Inga punctata (UGO 34), Macroptilium cirrhiferum (UGO 35), Rhynchosia americana (UGO 42), Dalea scandens (UGO 26), Dialum guianensis (UGO 35), Senna quinquangulata (UGO 38), S. reticulata (UGO 48) y Vigna adenantha (UGO 20).

En el análisis de agrupamiento local (Fig. 3), la menor similitud (0.35) arroja 2 grupos: las UGO septentrionales 1-33 y 37-38 y un segundo grupo conformado por las unidades más meridionales (UGO 34-36 y 39-48). En el diagrama de similitud regional (Fig. 4), con un valor de similitud muy bajo ( $0.16 \%)$, se distinguen 6 grupos: Chiapas, Aguascalientes, Miami-Dade, todas las tierras insulares (antillanas), Panamá, Chiapas y las UGO correspondientes a la Península de Yucatán.

Análisis de parsimonia de endemicidad (PAE) local y regional. Los PAE local y regional muestran resultados congruentes pero no idénticos a los obtenidos en el análisis de similitud (Figs. 3, 4). El análisis local excluyó 28 especies no informativas (de un total de 224) y arrojó 21 árboles igualmente parsimoniosos con una longitud de 982 pasos, un índice de consistencia de 19 y un índice de retención de 58. El árbol de consenso estricto local (Fig. 5) muestra una topología tipo escalera en la base, comenzando con las UGO 45 y 41 y posteriormente otras UGO meridionales, incluyendo un clado, correspondientes al extremo suroccidental de la Península de Yucatán (UGO 39, 40, 46, 47 y 48). Luego, hay una politomía con las UGO septentrionales (UGO 1-33). El árbol de consenso estricto regional (Fig. 6) muestra como grupo basal a Aguascalientes, posteriormente a Miami-Dade y luego una tricotomía que incluye las islas Caimán, el resto del Caribe insular
(Bahamas, Jamaica y Puerto Rico) y por otro lado las unidades operativas de América Central. En este último clado, la Península de Yucatán es el grupo hermano del clado que incluye Guatemala, Belice, Chiapas y Tabasco.

Análisis de distribución y trazos simplificados. La lista de leguminosas para la Península de Yucatán y su distribución global se presenta en el Apéndice; en la figura 7, un análisis de patrones de distribución de las 224 especies; 15 de ellas se distribuyen únicamente en la península (incluyendo el norte de Belice y Guatemala, Apéndice), lo que representa el $6.7 \%$ de las leguminosas. Otras especies previamente consideradas endémicas (Morrone, 2001, 2005), como Pithecellobium lanceolatum y P. graciflorum (= Cojoba graciflorum) presentan una distribución más amplia. En el otro extremo, se encuentran 90 especies de amplia distribución (pantropicales y neotropicales) y que no revelan relación alguna entre áreas. La Península de Yucatán comparte el mayor número de especies con Centroamérica (32) y América del Norte (11); le siguen América del Sur (8) y las Antillas (1) (Cuadro 3).

Análisis de filogenias de grupos selectos. Las filogenias evaluadas pertenecen a los géneros Desmanthus, Leucaena, Platymiscium y Swartzia, así como a las tribus Robinieae y Sesbanieae. La figura 8 resume la evolución de ocupación de áreas del género Desmanthus, un género originario del hemisferio norte (Norte América y México), mientras que la 9 y la 10 resumen la de los géneros Platymiscium y Swartzia de origen sudamericano.

Cuadro 3. Riqueza de especies por región biogeográfica y especies compartidas con la porción mexicana de la Península de Yucatán

\begin{tabular}{lcc}
\hline Región biogeográfica & \multicolumn{2}{c}{ Número de especies } \\
& Total & Compartidas \\
\hline Centro América & $90(\%)$ & $32(\%)$ \\
Sur América & 55 & 8 \\
Norte América & 43 & 11 \\
Península de Yucatán & 15 & 0 \\
Antillas & 21 & 1 \\
Neotropicales & 14 & 0 \\
Pantropicales & 76 & 0 \\
\hline
\end{tabular}




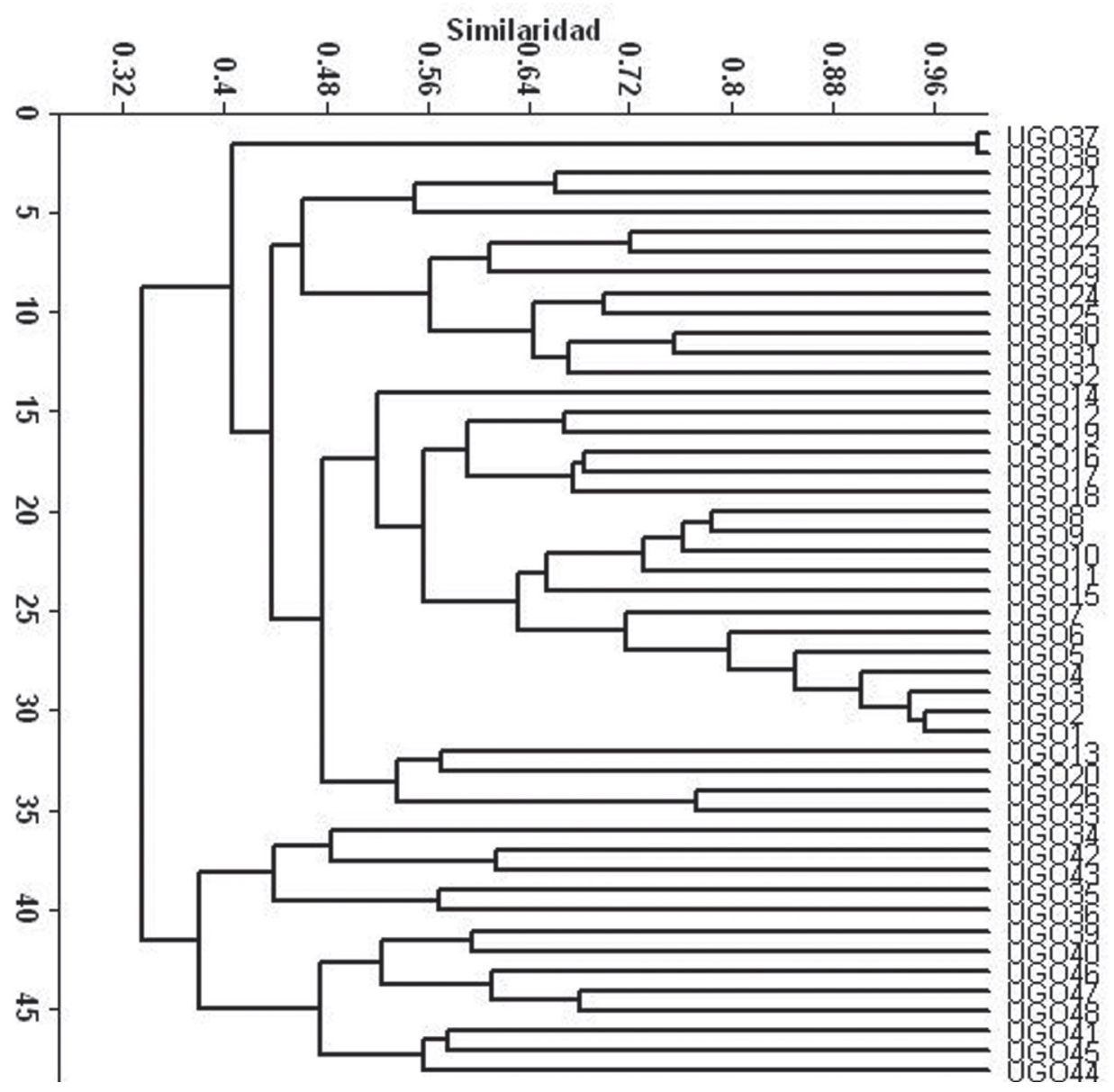

Figura 3. Similitud florística de las 48 unidades geográficas operativas (UGO) de la Península de Yucatán con base en la riqueza total de especies de leguminosas (análisis local). El grupo superior en la gráfica corresponde a las UGO 1-33 de las franjas más septentrionales, más las UGO 37 y 38; el grupo inferior corresponde a las UGO 34-48 de las franjas más meridionales (menos 37 y 38). Coeficiente de similitud utilizado: Jaccard; método de agrupamiento: UPGMA.

\section{Discusión}

La riqueza de leguminosas en la porción mexicana de la Península de Yucatán presentó una distribución heterogénea con valores más elevados al sur. Este aumento está documentado para otros organismos: helechos (RamírezBarahona et al., 2009), bromelias (Ramírez, com. pers.), orquídeas (Carnevali et al., 2001), árboles (Ibarra-Manríquez et al., 1995, 2002) y mamíferos (Simpson, 1964, Vázquez-Domínguez y Arita, 2010). Llaman la atención con los máximos valores de riqueza de especies, las UGO 37 y 38 correspondientes a la reserva de la Biosfera de Calakmul. No obstante, este resultado puede estar ligeramente influido por el hecho de que los datos de herbario fueron complementados con el listado florístico de Martínez et al. (2001) que incluye información para un área de $21 \mathrm{mil} \mathrm{km} \mathrm{km}^{2}$ lo cual muy posiblemente sobreestima la diversidad de estas UGO en relación con el resto de las unidades operativas con sólo 3 mil $\mathrm{km}^{2}$ y cuyo valor de riqueza proviene de registros de herbario.

En el caso opuesto, las áreas menos diversas (UGO 9 y 10) no se encuentran en la franja latitudinal más septentrional como se esperaría. Es posible que las UGO 1-6, caracterizadas por elementos típicos de la selva baja caducifolia y de la selva baja caducifolia con cactáceas columnares, presentes en todo el norte y centro de la región, esté enriquecida por elementos costeros distintivos del matorral de duna costera y la vegetación halófita. Otro elemento a tomar en cuenta es la intensa y prolongada transformación antrópica de las UGO 9 y 10 que puede eliminar especies raras $\mathrm{y} / \mathrm{o}$ sensibles.

Al menos 20 leguminosas están ampliamente distribuidas en la Península de Yucatán (presentes al menos en el $90 \%$ de las UGO) y corresponden a especies comunes en la selva baja caducifolia, vegetación secundaria, o bien, presentan una estrategia ruderal. Las legumino- 


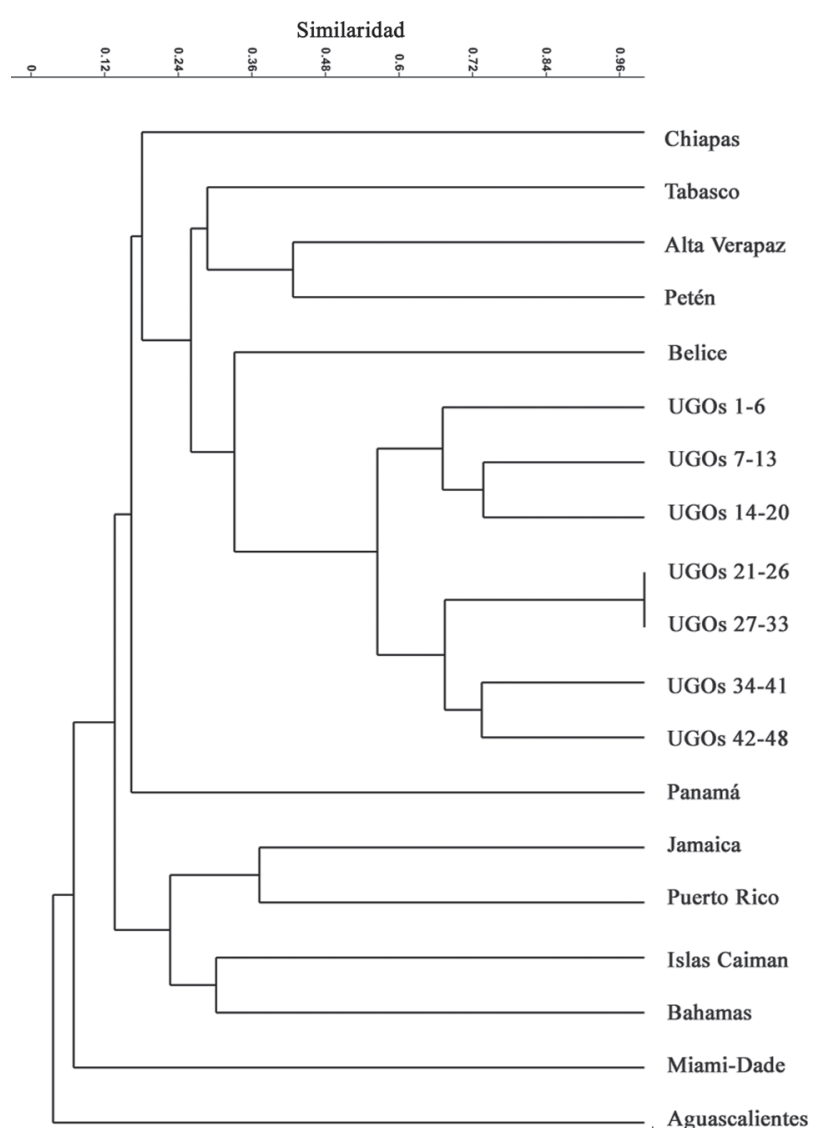

Figura 4. Similitud florística regional entre las 7 bandas geográficas operativas (UGO) de la Península de Yucatán y unidades geográficas operativas, escogidas de México, Centro América, Norte América, Sur América y el Caribe insular con base en la riqueza total de especies de leguminosas.

sas endémicas de la península son 15 (Carnevali et al. 2010; Apéndice) y en general son de amplia distribución en toda la región (varias UGO). Las especies que en la base de datos están presentes en una sola UGO, básicamente corresponden a especies típicas de otras provincias biogeográficas que en la Península están presentes de manera marginal, en especial en las franjas más meridionales. Este patrón ya se ha destacado para el caso de Souroubea loczyi (V.A. Richht.) De Roon (Marcgraviaceae) que crece en el área de la laguna de Términos (Endañú-Huerta et al., 2012).

En relación con los análisis de similitud florística local y regional, la porción mexicana de la península conforma la unidad biogeográfica Península de Yucatán, y esto lo confirma el análisis regional, al incluir todas las UGO de los 3 estados mexicanos como un grupo (Fig. 4). En el análisis local se observa una clara diferencia entre las UGO correspondientes a las bandas latitudinales más septentrionales y secas, y las más meridionales y húmedas, que se

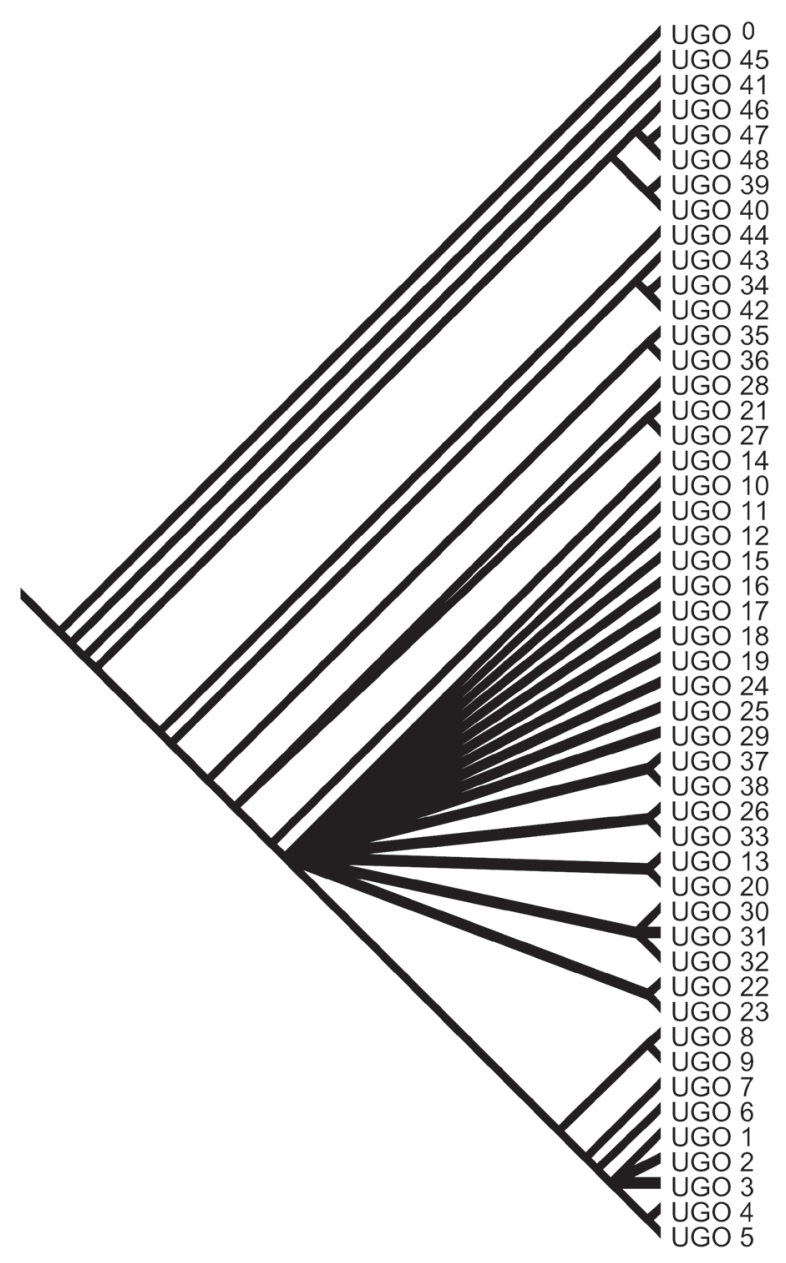

Figura 5. Árbol de consenso estricto de las relaciones florísticas de la Península de Yucatán (48 unidades operativas) basada en la distribución de 224 especies de leguminosas (PAE local). El árbol de consenso estricto fue obtenido a partir de 21 árboles más parsimoniosos (L: 989 pasos, IC: 19 y IR: 58).

refleja en un importante recambio de especies entre ambas áreas; por ejemplo, Diphysa Jacq. que tiene 3 especies en la Península de Yucatán; 2 en la región norte (D. carthagenensis y D. yucatanensis) y 1 en la región más meridional (D. paucifoliolata). Algo similar ocurre con el género Pithecellobium Mart., cuyas especies P. keyense, P. seleri y $P$. unguis-cati crecen en la región más septentrional, mientras que $P$. lanceolatum Benth. y $P$. winzerlingii Britton et Rose en la más meridional. Estos recambios también son evidentes en el nivel genérico: Dalea L., Marina Liebm.y Prosopis L. crecen en el norte, mientras que en la región sur se encuentran Ateleia (DC.) Benth., Calliandra Benth., Calopogonium Desv., Cojoba Britton et Rose, Cynometra L., Dialium L., Hymenaea L., Inga Mill., Neptunia Lour., Pterocarpus Bergius, Vatairea Aubl. y Zygia P. Browne. Se 


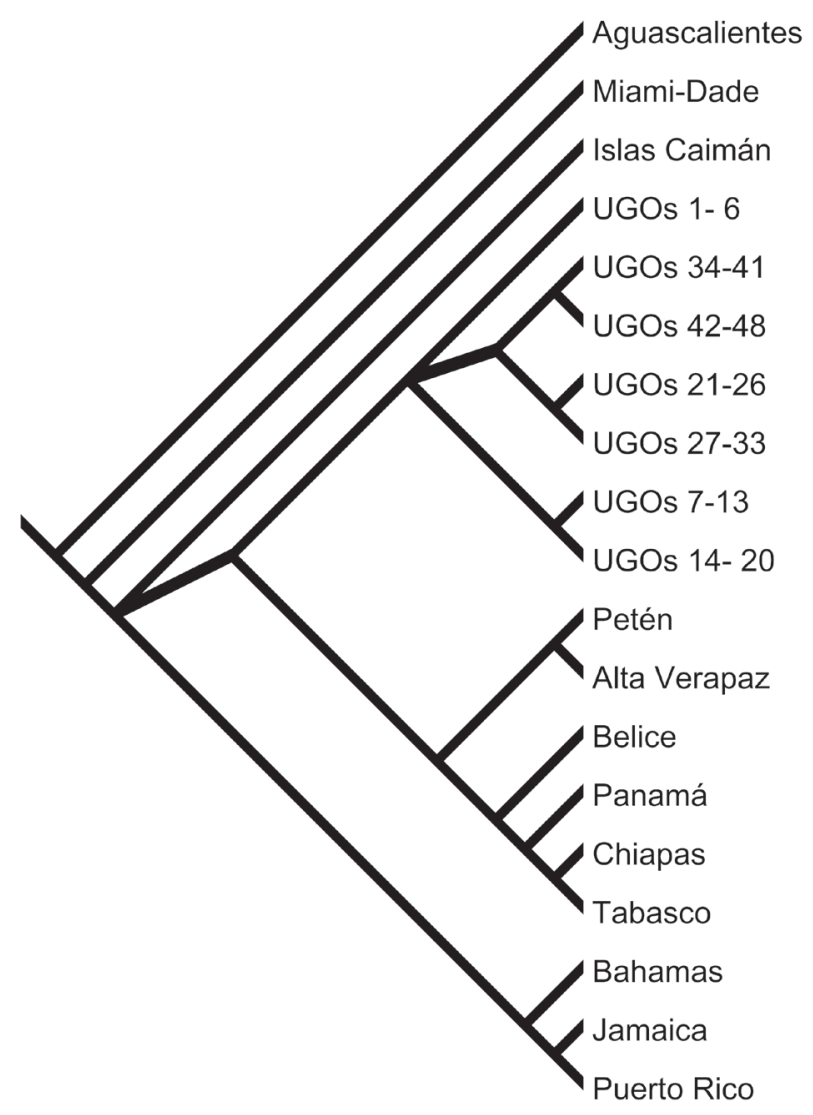

Figura 6. Árbol regional de consenso estricto de las relaciones florísticas las UGO de la Península de Yucatán y áreas de México, Centro América, Norte América, Sur América y el Caribe. La matriz incluye de 998 especies de leguminosas (PAE regional). El árbol de consenso se obtuvo a partir de 4 árboles más parsimoniosos (L: 1195 pasos, IC: 38 y IR: 54).

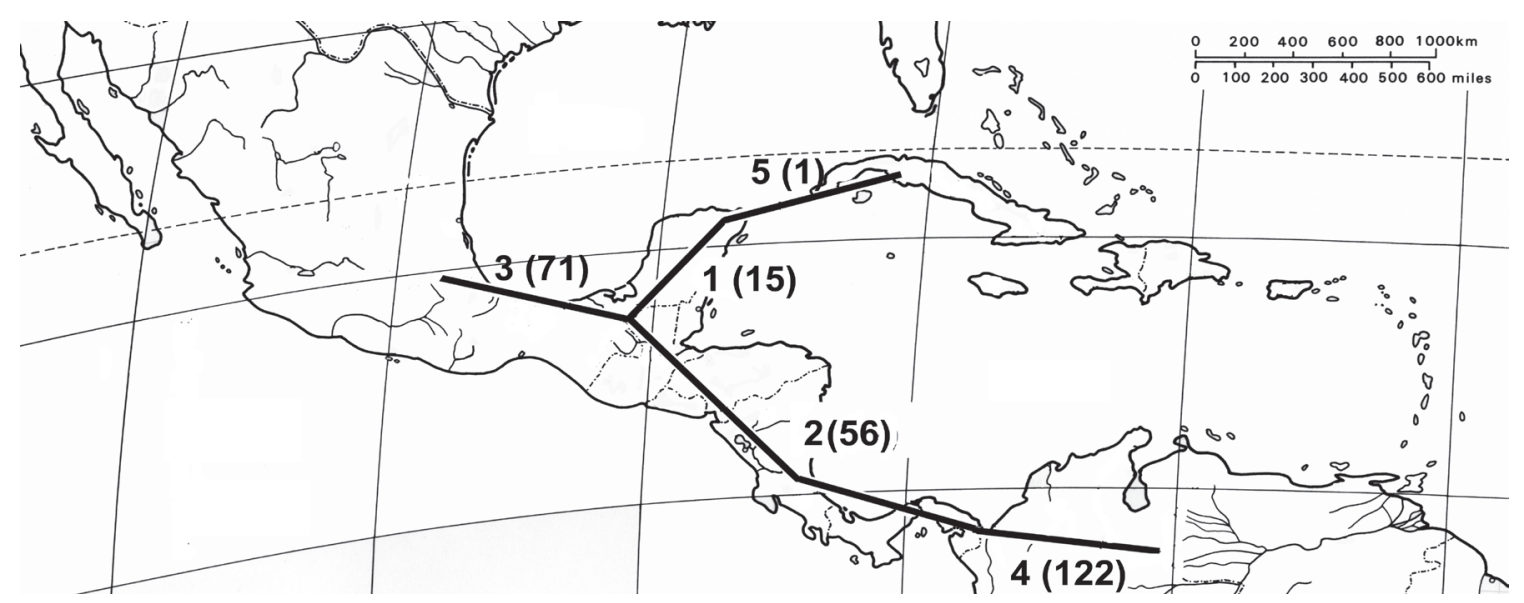

Figura 7. Patrones de trazos individuales observados. La mayoría de los trazos individuales tiene un sentido norte-sur. 1, taxones exclusivos de la Península de Yucatán (15); 2, Península de Yucatán y Centro América (56); Península de Yucatán, Centro América y Norte América (71); Península de Yucatán, Centro América, Norte América y Sur América (122); Península de Yucatán y las Antillas (1). encontraron distribuciones análogas por Ibarra-Manríquez et al. (2002) en el caso de árboles (Fig. 5).

La separación entre las 5 franjas latitudinales más septentrionales y las 2 más meridionales coincide con la clasificación de Smith (1941) basada en las especies de lagartijas del género Sceloporus; mejor aún, con la de unidades morfotectónicas de la plataforma de Yucatán (Arita y Vásquez-Domínguez, 2003) y en menor grado con la propuesta de Goldman y Moore (1945) ya que la línea que separa ambas unidades se desplaza en la porción oriental más hacia el norte. Estas 2 unidades corresponden a lo que varios autores han llamado Provincia o Distrito Yucatán y Provincia y Distrito Petén respectivamente (Smith, 1991; Arriaga et al., 1997; Ramírez-Barahona, 2009). Los análisis de similitud y PAE apoyan la distinción de la Península de Yucatán como una Provincia y 2 subunidades (distritos). Una subdivisión más fina (subdistritos) permite reconocer 3 unidades en el norte y 2 en el sur (Fig. 11). Las subunidades se disponen en bandas latitudinales con la excepción de una franja oriental que coincide bastante bien con la unidad geomorfológica planicie marina holocénica (LugoHubo et al., 1992) y con el subdistrito de Sian Ka'an de Ramírez-Barahona (2009). Los 5 distritos propuestos son distintos a propuestas previas (véase Ibarra-Manríquez et al. 2002, Ramírez-Barahona, 2009). En este sentido, una clasificación en subdistritos posiblemente requiera una evaluación global de variables abióticas y diversos grupos de organismos.

El análisis de similitud regional presenta una notable estructura geográfica (Fig. 4): las UGO de América Central, las del Caribe insular y las de Norte América (Aguascalientes y Miami). Varios autores incluyen los departamentos 


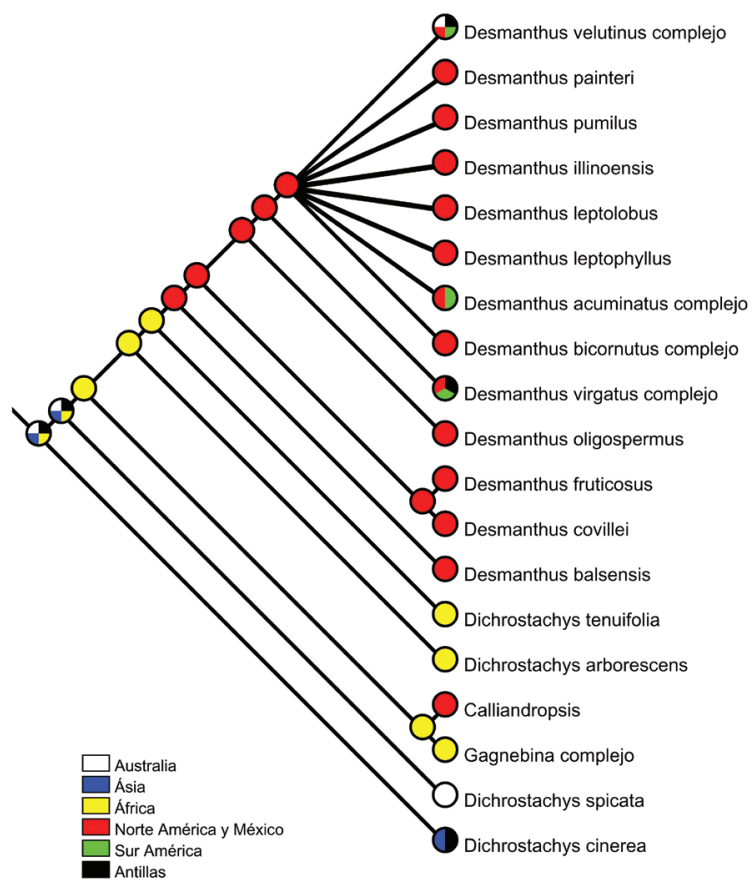

Figura 8. Filogenia (árbol de consenso estricto) y mapeo de áreas de ocupación de taxones terminales y nodos del género Desmanthus (tomado con modificaciones de Luckow 1993).

Corozal y Orange Walk de Belice y El Petén de Guatemala, como parte de esta provincia biogeográfica (e.j. EstradaLoera, 1991) y este análisis apoya al menos la inclusión de Belice y en menor medida la del Petén guatemalteco. Las diferencias en el tamaño de las unidades operativas y el grado de conocimiento de las áreas (Cuadro 2) pueden influir en las de comparaciones de similitud.

El análisis de parsimonia de endemicidad (PAE) local (Fig. 5) muestra resultados congruentes (estructura geográfica) pero no idénticos con el análisis de similitud local. Una topología de escalera para las UGO meridionales y un clado con una politomía que incluye las más septentrionales. La topología del árbol muestra estrecha relación con un gradiente latitudinal; áreas más meridionales en la base y progresivamente áreas más septentrionales en las ramas terminales. En el caso del PAE regional (Fig. 6), los resultados son también congruentes con el análisis de similitud regional, pero se pierde parte de la estructura geográfica. Aguascalientes y Miami (Norte América) aparecen como unidades basales con muy pocas especies compartidas con el resto de las UGO; posteriormente, se obtiene un clado caribeño (excepto las islas Caimán) y un clado continental, que incluye a su vez las unidades operativas de México (Tabasco y Chipas) y el resto de Centro América, y otro clado representado por todas las UGO de la porción mexicana de la Península de Yucatán. Este análisis no apoya la inclusión de Belice y el Petén guatemalteco como parte de la Península de Yucatán. Sin embargo, es posible que un análisis futuro con áreas del mismo tamaño y la misma calidad de información (herbario vs. listados florísticos) modifique la topología de este clado continental.

En relación con la distribución general de las 224 especies y los trazos individuales simplificados, 88 especies presentan una amplia distribución (pantropicales y/o neotropicales) y no permiten reconocer relaciones entre áreas. Pero si es informativo el número de especies compartidas exclusivamente por 2 áreas. Los trazos simplificados más repetidos para las leguminosas son los dispuestos en el eje continental norte-sur (Fig. 7). En el primer caso, hay 15 especies con un trazo que incluye la Península de Yucatán más Guatemala y Belice (trazo 1), otras 41 especies se añaden si incluimos América Central (trazo 2), 15 más si incluimos América del Norte (trazo 3) y por último, 51 más hasta América del Sur (trazo 4). Sólo Ateleia cubensis tiene un trazo que incluye exclusivamente la Península de Yucatán y las Antillas (trazo 5). Lo anterior sugiere que la Península de Yucatán es una unidad biogeográfica continental que comparte más especies con América Central, América del Sur y América del Norte que con las Antillas (Fig. 7). Estos resultados coinciden con los de Vásquez-Domínguez y Arita (2010), quienes destacaron que, con pocas excepciones, la fauna de la región norte de la península es un subconjunto de la fauna de la base (región meridional) y que el intercambio entre Yucatán y 
las islas del Caribe, es mínimo. El aislamiento geográfico y la barrera a la dispersión que representa el canal de Yucatán pueden ser evaluados a través del endemismo genérico. Las Antillas cuentan con varios géneros endémicos de leguminosas; Arcoa Urb. (1 sp.), Behaimia Grisb. (2), Brya P. Browne (7), Hebestigma Urb. (2), Herpyza Sauvalle (1), Neorudolphia Britton (1), Pictetia DC (17), Poitea Vent. (13) y Rhodopis (2). Por otro lado, no hay géneros endémicos de leguminosas en la Península de Yucatán. Otras familias de angiospermas muestran patrones similares: las orquídeas presentan 13 géneros endémicos en las Antillas (Acevedo y Strong, actualización continua) y ninguno en la península (Carnevali et al., 2010). Las palmas (Arecaceae) también presentan un patrón de distribución que apoya una mayor afinidad continental; de los 14 géneros presentes, 10 son de origen continental ( 9 centroamericanos y 1 norteamericano) y 4 de origen antillano (Quero, 1989).

Las filogenias evaluadas para varios géneros de leguminosas muestran un patrón biogeográfico que sugiere origen tropical proveniente del hemisferio norte y en segundo lugar del hemisferio sur; pero ninguno hasta ahora del Caribe insular. El género Desmanthus (Fig. 8), con 24 especies, presenta su mayor diversidad en el SE de los Estados Unidos de América y México y sólo 2 especies exclusivamente suramericanas y antillanas, y 4 distribuidas en ambos hemisferios, ubicadas en posiciones derivadas del árbol (Luckow, 1993, 1997). Otro género, Leucaena con 22 especies, presenta su mayor diversidad en el norte y centro de México, un segundo centro de distribución se ubica al sur del istmo de Tehuantepec, pero sólo 1 especie se encuentra en América del Sur y 2 en las Antillas (adventicias) y en posiciones derivadas del árbol (Hughes, 1998; Hughes et al., 2002). Otros géneros presentes en la Península de Yucatán con patrones de distribución similares son: Coursetia DC, Gliricida Kunth, Lennea Klotsch (tribu Robinieae), Sesbania Adans. (tribu Sesbanieae) (Lavin y Sousa, 1995), Dalea L. y Marina L. (tribu Amorphae) (Mcmahon y Hufford, 2004). También existen géneros originarios del hemisferio sur que se han desplazado hasta México y América del Norte. El género Swartzia (Fig. 9) muestra un clado basal (bentamioide) exclusivamente suramericano, mientras que $S$. cubensis, única especie presente en la península y otras especies centroamericanas están incluidas en clados terminales (Torke y Schaal, 2008). De igual manera, Platymiscium (Fig. 10), con 19 especies, presenta 2 centros de diversidad, uno en Brasil y otro en México; la filogenia sugiere un origen en áreas secas suramericanas y su posterior radiación hacia el norte, el clado más derivado sólo incluye especies centroamericanas y mexicanas (Saslis-Lagoudakis et al., 2008). Estas filogenias apoyan historias evolutivas restringidas al continente americano y en menor grado relacionadas con las Antillas.

En resumen, los resultados sugieren que la Península de Yucatán, a pesar de compartir la misma banda climática con las Antillas y geomorfología similar, tiene más elementos comunes con floras de otras latitudes continentales (e.g. Centroamérica). Así que una fuerte barrera a la dispersión de taxones del este al oeste debe invocarse como explicación. Los resultados apoyan la propuesta de Estrada-Loera (1991), Ibarra- Manríquez et al. (2002), Ramírez-Barahona et al. (2009) y Vásquez-Domínguez y Arita (2010) de una mayor afinidad continental, en contradicción con aquellos trabajos donde se destaca una mayor relación con las Antillas (Delgadillo, 1984; Morone, 2006).

Por otra parte, los patrones de regionalización basados en la distribución de leguminosas coinciden con los obtenidos para lagartijas del género Sceloporus (Smith, 1941) y para plantas endémicas (Espadas Manrique et al., 2003) que encuentran mayor riqueza de especies en la porción sur, y que sugiere una segunda barrera a la dispersión de especies, posiblemente climática, entre el norte seco conformado por las 5 franjas más septentrionales (UGO 1-33, Distrito Yucatán) y las 2 franjas más meridionales (UGO 34-48, Distrito Petén). Una clasificación más fina reconoce al menos 5 subunidades (Fig. 11). Los resultados también apoyan la propuesta biogeográfica de Dinersten et al. (1995); en este sentido, al seguir los esquemas biogeográficos de Morrone (2005) para México, es recomendable transferir la Península de Yucatán del Dominio Caribe al Dominio Mesoamericano.

\section{Agradecimientos}

A CONABIO y a los siguientes proyectos, responsables e instituciones: AC 3 Computarización de la colección de plantas acuáticas mexicanas del Herbario Nacional (MEXU), 2a. etapa, A. Novelo Retana, MEXU; AE 13 Catálogo electrónico de especímenes depositados en el Herbario de la Universidad de Texas en Austin, Fase IV, J. L. Panero, Herbarium of the The University of Texas; B 140 Actualización de las bases de datos de colecciones, especies en peligro de extinción, colecta y propagación de Germoplasma, A. P. Vovides Papalouka, INECOL, A.C.; B 70 Distribución de las especies endémicas de la Península de Yucatán, R. Durán García, Herbario CICY; BC 6 Sistematización de la colección científica de flora del herbario Eizi Matuda, de la Universidad de Ciencias y Artes de Chiapas, M. Á. Pérez Farrera, Herbario Eizi Matuda, Universidad de Ciencias y Artes de Chiapas; BE 21 Flora y datos básicos para la evaluación de las actividades apícola y forestal en tres áreas focales del corredor Sian Ka'an-Calakmul, F. Chiang Cabrera, MEXU; BE 


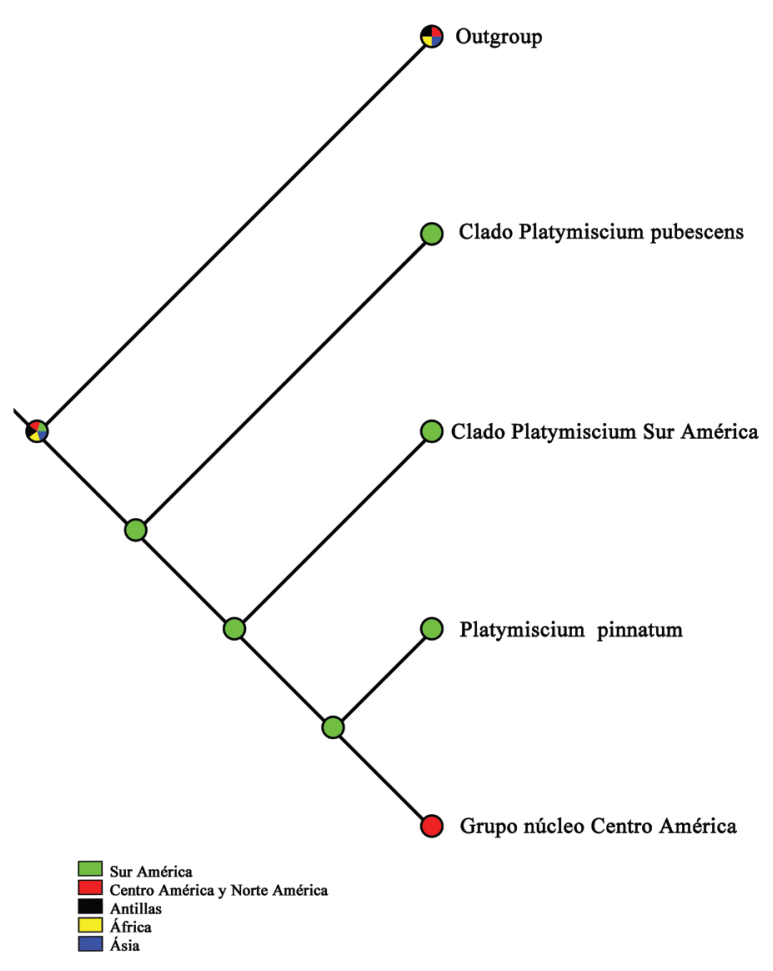

Figura 9. Filogenia (árbol de consenso estricto) y mapeo de áreas de ocupación de taxones terminales y nodos del género Platymiscium (tomado con modificaciones de Saslis-Lagoudakis et al., 2008).

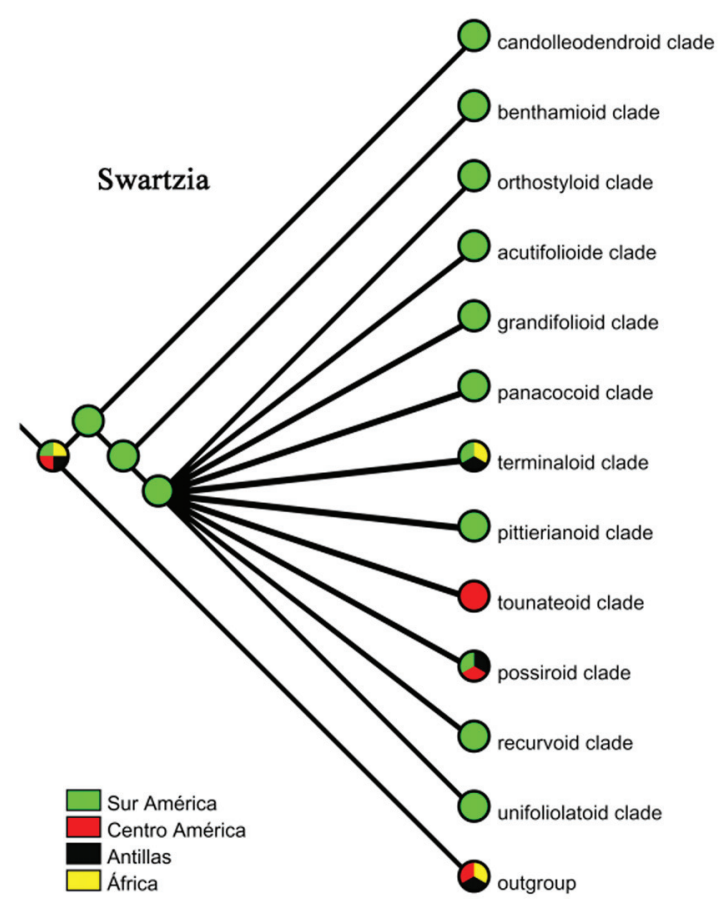

Figura 10. Filogenia (árbol de consenso estricto) y mapeo de áreas de ocupación de taxones terminales y nodos del género Swartzia Schreb. (tomado con modificaciones de Torke y Schaal, 2008). 


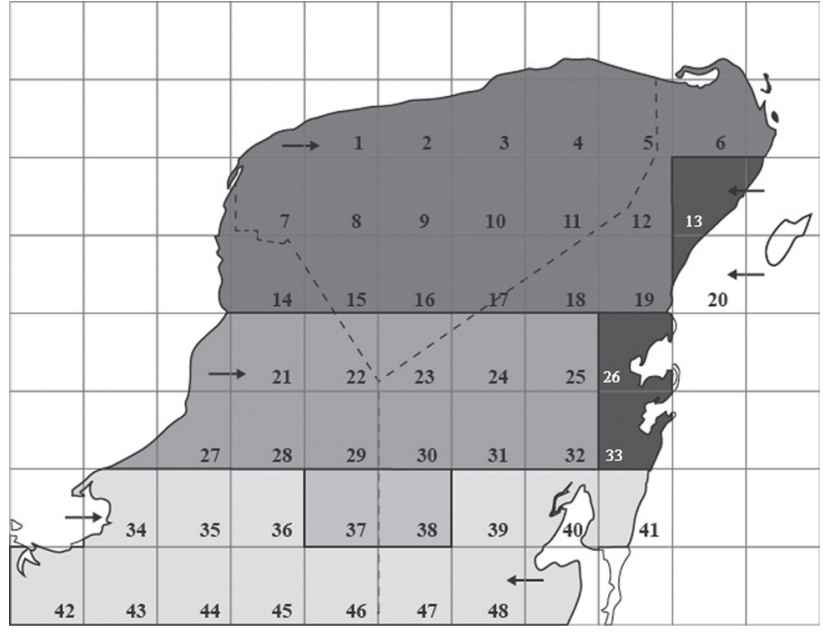

Figura 11. Esquema de regionalización de la porción mexicana de la Península de Yucatán basada en el análisis de similaridad de leguminosas.

23 Computarización de la colección de plantas acuáticas mexicanas del Herbario Nacional (MEXU), 3a etapa, A. Novelo Retana, Instituto de Biología, UNAM; BE 7 La familia Asteraceae en México, fase IV, J. L. Villaseñor Ríos, Instituto de Biología, UNAM; BJ 2 Uso y monitoreo de los recursos naturales en el Corredor Biológico Mesoamericano (áreas focales Xpujil-Zoh Laguna y Carrillo Puerto), Pozo de la Tijera, El Colegio de la Frontera Sur; BK 31 Flora acuática vascular del área de protección de flora y fauna Laguna de Términos, Campeche, México, Jaime Raúl Bonilla Barbosa, Laboratorio de Hidrobotánica Universidad Autónoma del Estado de Morelos; H 146 Actualización del banco de datos florístico de la Península de Yucatán, S. Flores Guido, UADY; J 1 Propuesta para sistematizar la colección palinológica de polen reciente y fósil del IGLUNAM, E. Martínez Hernández, Departamento de Paleontología, UNAM; J 10 Catálogos florísticos de México por entidad federativa e información etnobotánica de la Colección del Herbario Nacional, L. Vela Gálvez, INIF y M. V. Gutiérrez Garduño, Herbario Nacional Forestal; J 2 Inventario nacional de especies vegetales y animales de uso artesanal, C. B. Marentes, Asociación Mexicana de Arte y Cultura Popular, A.C.; J 63 Sistema de apoyo a la toma de decisiones para la reforestación rural en México, D. D. Reygadas Prado, Instituto Nacional de Investigaciones Forestales Agrícolas y Secretaría de Agricultura, Ganadería y Desarrollo Rural; J 84 Árboles mexicanos potencialmente valiosos para la restauración ecológica y la reforestación, Vázquez.Yanes, Laboratorio de Ecología Fisiológica, Instituto de Ecología, UNAM. En el Centro de Investigación Científica de Yucatán, agradecemos a Paola Marfil Lara, por las figuras, a Silvia Hernández. y al herbario CICY. Finalmente, a Guillermo
Ibarra Manríquez por sus comentarios, los cuales fueron de gran ayuda para mejorar el contenido del artículo.

\section{Literatura citada}

Acevedo, P. y M. Strong. [actualización continua]. Flora of the West Indies. http://persoon.si.edu/antilles/westindies/index. htm; última consulta: 15.0I.2012.

Arita, H. T. y E. Vásquez-Domínguez. 2003. Fauna y la conformación de la provincia biótica yucateca: biogeografía y macroecología. In Naturaleza y sociedad en el área maya: pasado, presente y futuro, P. Colunga-García Marín y A. Larqué Saavedra, eds.). Centro de Investigación Científica de Yucatán, Mérida Yucatán. p. 69-80.

Arriaga, L., C. Aguilar, D. Espinosa-Organista y R. Jiménez. 1997. Regionalización ecológica y biogeográfica de México. Comisión Nacional para el Conocimiento y Uso de la Biodiversidad (CONABIO), México, D. F. 55 p.

Balick, M. J., M. H. Nee y D. E. Atha. 2000. Checklist of the vascular plants of Belize. Memories of the New York Botanical Garden 85:i-ix, 1-246.

Balleza, J. de J., J. L. Villaseñor y G. Ibarra-Manríquez. 2005. Regionalización biogeográfica de Zacatecas, México, con base en los patrones de distribución de la familia Asteraceae. Revista Mexicana de Biodiversad 76:71-78.

Barrera, A. 1962. La península de Yucatán como provincia biótica. Revista de la Sociedad Mexicana de Historia Natural 23:71105.

Birks, H. J. B. 1987. Recent methodological developments in quantitative descriptive biogeography. Annales Zoologici Fennici 24:165-178.

Breedlove, D. E. 1986. Listados Florísticos de México. IV. Flora de Chiapas. Instituto de Biología, UNAM, México, D. F. 246 p.

Carnevali, G., J. L. Tapia-Muñoz, R. Duno-de Stefano e I. Ramírez Morillo. 2010. Flora ilustrada de la península de Yucatán. Listado florístico. Centro de Investigación Científica de Yucatán, Mérida, Yucatán. 326 p.

Carnevali, G., J. L. Tapia-Muñoz, R. Jiménez-Machorro, L. Sánchez-Saldaña, L. Ibarra-González, I. M. Ramírez y M. P. Gómez-Juárez. 2001. Notes on the flora of the Yucatán Península II: a synopsis of the orchid flora of the Mexican Yucatán Península and a tentative checklist of the Orchidaceae of the Yucatán Península Biotic Province. Harvard Papers in Botany 5:383-466.

Chiappy-Jhones, C., V. Rico-Gray, L. Gama y L. Giddings. 2001. Floristic affinities between the Yucatán Península and some karstic areas of Cuba. Journal of Biogeography 28:535-542.

Correa, M. D., C. Galdames y M. Sánchez de Stapf. 2004. Catálogo de las plantas vasculares de Panamá. Universidad de Panamá, Instituto de Investigaciones Tropicales, Autoridad Nacional del Ambiente, Corredor Biológico Mesoamericano del Atlántico Panameño, Ciudad de Panamá, 
Panamá. Croizat, L. 1958. Panbiogeography, volúmenes. 1 y 2. Publicado por el autor. Caracas. $1731 \mathrm{p}$.

Crovello, T. J. 1981. Quantitative biogeography: an overview. Taxon 30:563-575.

Delgado, M. E. 1996. Leguminosas de Aguascalientes. Universidad Autónoma de Aguascalientes, Aguascalientes. 193 p.

Delgadillo, M. C. 1984. Mosses of the Yucatán Península, Mexico. III. Phytogeography. Bryologist 87:12-16.

Dinerstein, E., D. Olson, D. Graham, A. Webster, S. Primm, M. Bookbinder y G. Ledec 1995. Una evaluación del estado de conservación de las ecorregiones terrestres de América Latina y el Caribe. World Bank-Fondo Mundial para la Naturaleza, Washington, D.C. 135 p.

Durán, R., e I. Olmsted. 1997. Listado florístico de la Reserva de Sian Ka'an. Amigos de Sian Ka'an. Puerto Morelos, Quintana Roo. 71 p.

Durán, R., P. Sima y M. Juan-Qui. 1999. Listado florístico de Ría Celestún. Centro de Investigación Científica de Yucatán, Mérida, Yucatán. s/f.

Endañú Huerta, E., J. E. López Contreras, R. Duno-de Stefano y G. Carnevali Fernández-Concha. 2012. Souroubea loczyi (Marcgraviaceae) un registro nuevo para la Península de Yucatán. Polibotánica 33:33-39.

Espejel, I. 1987. A phytogeographical analysys of costal vegetation in the Yucatán Península. Journal of Biogeography 14:499-519.

Estrada-Loera, E. 1991. Phytogeographic Relationships of the Yucatán Península. Journal of Biogeography 19:687-697.

Ferrusquía-Villafranca, I. 1993. Geology of Mexico: a synopsis. In Biological diversity of Mexico: origins and distribution, T. P. Ramamoorthy, R. Bye, A. Lot y J. Fa (eds.). Oxford University Press, New York. p. 3-107.

Flores, J. S. 2001. Leguminosae. Florística, etnobotánica y ecología. Etnoflora Yucatanense, Fascículo 18. Universidad Autónoma de Yucatán, Mérida, Yucatán. 320 p.

Goldman, E. A. y R. T. Moore. 1945. The biotic provinces of Mexico. Journal of Mammology 26:347-361.

Goloboff, P. A. 1993. NONA, versión 2.0. Publicado por el autor. Tucumán.

Hammer, Ø., D. A. T. Harper y P. D. Ryan. 2001. PAST: Paleontological statistics software package for education and data analysis. Palaeontología Electrónica 4. http://palaeoelectronica.org/2001_1/past/issue1_01.htm; última consulta: 15.0I.2012.

Hubp, J. L. y Ma. T. García-Arizaga. 1999. Geomorfología. In Atlas de procesos territoriales de Yucatán, A. García de Fuentes, C. Córdoba y Ordóñez y P. Ponce de León (eds.). Facultad de Arquitectura, Universidad Autónoma de Yucatán, Mérida. 158-162 p.

Hughes, C. E. 1998. Monograph of Leucaena (LeguminosaeMimosoideae). Systematic Botany Monographs 55:1-244.

Hughes, C. E., C. Donovan-Bailey y S. A. Harris. 2002. Divergent and reticulate species relationships in Leucaena (Fabaceae) inferred from multiple data sources: insights into polyploid origins and nrDNA polymorphism. American Journal of Botany 89: 1057-1073.

Ibarra-Manríquez, G., J. L.Villaseñor y R. Durán. 1995. Riqueza de especies y endemismo del componente arbóreo de la Península de Yucatán, México. Boletín de la Sociedad Botánica de México 57:49-77.

Ibarra-Manríquez, G., J. L. Villaseñor, R. Durán y J. Meave. 2002. Biogeographical analysis of the tree flora of the Yucatán Península. Journal of Biogeography 29:17-29.

Jardine, N. 1972. Computational methods in plant distributions. In Taxonomy, phytogeography and evolution, D. H. Valentine (ed.). Academic Press, London. p. 381-393.

Lavin, M. y M. Sousa. 1995. Phylogenetic systematics and biogeography of the tribe Robinieae. Systematic Botany Monographs 45:1-165.

Lee, J. C. 1980. An ecogeographic analysis of the herpetofauna of the Yucatan Peninsula. Miscellaneous publication Museum of Natural History, University of Kansas 67:1-75.

Luckow, M. 1993. Monograph of Desmanthus (LeguminosaeMimosoideae). Systematic Botany Monographs 38:1-166.

Luckow, M. 1997. Generic relationships in the Dichrostachys group (Leguminosae: Mimosoideae): evidence from chloroplast DNA restriction sites and morphology. Systematic Botany 22:189-198.

Luckow, M. y M. Lavín. 1993. Origins and relationships of tropical North America in the context of the boreotropics hypothesis. American Journal of Botany 80:1-14.

Lundell, C. L. 1934. Preliminary sketch of the phytogeography of the Yucatán Peninsula. Contributions to American Archaeology 12:257-321.

Maddison, W. P. y D. R. Maddison. 2000. Mesquite: a modular system for evolutionary analysis, ver. $2.75 \mathrm{http}: / /$ mesquiteproject.org; última consulta: 15.0I.2012.

Martínez, E., M. Sousa y C. H. Ramos-Álvarez. 2001. Listados Florísticos de México. XXII. Región de Calakmul, Campeche. Universidad Nacional Autónoma de México, México, D. F. 55 p.

McMahon, M. y L. Hufford. 2004. Phylogeny of Amorpheae (Fabaceae: Papilionoideae). American Journal of Botany 91:1219-1230.

Miranda, F. 1958. Rasgos fisiográficos de interés para estudios botánicos. In Los recursos naturales del Sureste y su aprovechamiento II, E. Beltrán (ed.). Instituto Mexicano Recursos Naturales Renovables, México, D. F. p. 215-271.

Morrone, J. J. 2001. Biogeografía de América Latina y el Caribe. Manuales y Tesis SEA 3, SEA, Zaragoza. 148 p.

Morrone, J. J. 2005. Hacia una síntesis biogeográfica de México. Revista Mexicana de Biodiversidad 76:207-252.

Morrone, J. 2006. Biogeographic areas and transition zones of Latin America and the Caribbean islands based on panbiogeographic 
and cladistic analysis of the Entomofauna. Annual Review of Entomology 51:467-94.

Murguía, M. y F. Rojas. 2001. Biogeografía cuantitativa. In Introducción a la biogeografía en Latinoamérica: teorías, conceptos, métodos y aplicaciones, J. Llorente y J. J. Morrone (eds.). Universidad Nacional Autónoma de México, México, D. F. p.39-47.

Nixon, K. C. 1999. Winclada (beta) ver. 0.9.9. Publicado por el autor, Ithaca. $11 \mathrm{p}$.

Olmsted, I., R. Durán, J. A. González-Iturbe, J. Granados y F. Tun. 1999. Vegetación. In Atlas de procesos territoriales de Yucatán, García, A. y J. Córdoba y Ordóñez (eds.). Facultad de Arquitectura, Universidad Autónoma de Yucatán, Mérida. $255 \mathrm{p}$.

Orellana R., M. Balam e I. Bañuelos (coords.). 1999. Evaluación climática (climatología de la Península de Yucatán). In Atlas de procesos territoriales de Yucatán, A. García de Fuentes, C. Córdoba y Ordoñez y P. Ponce de León (eds.). Facultad de Arquitectura, Universidad Autónoma de Yucatán, Mérida. p. 183-194.

Pérez A. L., M. Sousa, A. M. Hanan, F.Chiang y P. Tenorio. 2005. Vegetación terrestre. In Biodiversidad del estado de Tabasco, J. Bueno, F. Álvarez y S. Santiago (eds.). Instituto de Biología, UNAM/ CONABIO, México, D. F. p. 65-110.

Quero, H. J. 1989. Flora genérica de Arecaceae de México. Tesis, doctorado Facultad de Ciencias, Universidad Nacional Autónoma de México, México D. F. 142 p.

Ramírez-Barahona, S., A. Torres-Miranda, M. Palacios-Ríos e I. Luna-Vega. 2009. Historical biogeography of the Yucatán Península, Mexico: a perspective from ferns (Monilophyta) and lycopods (Lycophyta). Biological Journal of the Linnean Society 98:775-786.

Rzedowski, J. 1978. Vegetación de México. Limusa, México, D. F. $432 \mathrm{p}$.

Saslis-Lagoudakis, C., M. W. Chase, D. N. Robinson, S. J. Russell y B. B. Klitgaard. 2008. Phylogenetics of Neotropical Platymiscium (Leguminosae: Dalbergieae): systematics, divergence times, and biogeography inferred from nuclear ribosomal and plastid DNA sequence data. American Journal of Botany 95:1270-1286.

Schultz, G. P. 2005. Vascular flora of the El Edén Ecological
Reserve, Quintana Roo, Mexico. Journal of the Torrey Botanical Society 132:311-322.

Sousa, M. y A. Delgado. 1993. Mexican Leguminosae: phytogeography, endemism, and origins. In $\mathrm{T}$. Ramamoorthy, R. Bye y J. Fa (eds.). Biological diversity of México: origins and distribution. Oxford University.Press, New York. p. 459-513.

Simpson, G. G. 1964. Species densities of North American recent mammals. Systematic Zoology 13:57-73.

Smith, H. M. 1941. Las provincias bióticas de México, según la distribución geográfica de las lagartijas del género Sceloporus. Anales de la Escuela Nacional de Ciencias Biológicas 2:103-110.

Standley, P. y J. Steyermark. 1946. Leguminosae. Flora de Guatemala. Fieldiana Botany 24:244-245.

Torke, B. M. y B. A. Schaal. 2008. Molecular phylogenetics of the species-rich neotropical genus Swartzia (Leguminosae, Papilionoideae) and related genera of the swartzioid clade. American Journal of Botany 95:215-228.

Trejo-Torres, J. C. 2003. Biogeografía ecológica en las Antillas: ejemplos de las orquídeas y las selvas cársticas. In Una perspectiva Latinoamericana de la biogeografía, J. J. Morrone y J. Llorente-Bousquets (eds.). Universidad Nacional Autónoma de México, México D.F. p. 199-208.

Trejo-Torres, J. C. y J. D. Ackerman. 2001. Biogeography of the Antilles based on a parsimony analysis of orchid distributions. Journal of Biogeography 28:775-794.

Vázquez-Domínguez, E. y H. T. Arita. 2010. The Yucatán Península: biogeographical history 65 million years in the making. Ecography 33:212-219.

White, D. A. y S. P. Darwin. 1995. Woody vegetation of tropical lowland deciduous forests and Mayan ruins in the northcentral Yucatán Península, Mexico. Tulane Studies in Zoology \& Botany 30:1-25.

White, D. A. y C. S. Hood. 2004. Vegetation patterns and environmental gradients in tropical dry forest of the northern Yucatán Península. Journal of Vegetation Science 15:151-160.

Wunderlin, R. P. y B. F. Hansen. 2008. Atlas of Florida vascular plants. http://www.plantatlas.usf.edu/; última consulta: 15.0I.2012. 
Apéndice. Especies de Leguminosae presentes en Península de Yucatán, indicándose el área de su distribución geográfica. Abreviaturas de las áreas: PAN, pantropical; NEO, neotropical; NA, Norte América; CA, Centro América; SA, Sur América; ANT, Antillas; GUA y BEL, Guatemala y Belice. Abreviaturas del hábito: a, árbol; ar, arbusto; h, hierba; su, sufrútice; th, trepadora herbácea; tl, trepadora leñosa. Se resaltan con gris las endémicas de la Provincia Biótica Península de Yucatán, que incluye áreas vecinas de Belice (Corozal y Orange Walk) y Guatemala (Petén).

\begin{tabular}{|c|c|c|c|c|c|c|c|c|}
\hline Especie & $P A N$ & $N E O$ & $N A$ & $C A$ & $S A$ & $A N T$ & GUA y $B E L$ & Hábito \\
\hline \multicolumn{9}{|l|}{ CAESALPINIOIDEAE } \\
\hline Bauhinia divaricata $\mathrm{L}$. & 0 & 0 & 1 & 1 & 0 & 1 & 0 & $\mathrm{a} / \mathrm{ar}$ \\
\hline B. erythrocaly Wunderlin & 0 & 0 & 0 & 0 & 0 & 0 & 1 & $\mathrm{a} / \mathrm{ar}$ \\
\hline B. herrerae (Britton et Rose) Standl. et Steyerm. & 0 & 0 & 0 & 1 & 1 & 0 & 0 & $\mathrm{tl}$ \\
\hline B. jenningsii P. Wilson & 0 & 0 & 0 & 1 & 0 & 1 & 0 & $\mathrm{ar} / \mathrm{tl}$ \\
\hline B. ungulata $\mathrm{L}$. & 0 & 0 & 1 & 1 & 1 & 0 & 0 & $\mathrm{a}$ \\
\hline Caesalpinia bonduc (L.) Roxb. & 1 & 0 & 0 & 0 & 0 & 0 & 0 & $\mathrm{a}$ \\
\hline C. gaumeri Greenm. & 0 & 0 & 0 & 0 & 0 & 0 & 1 & $\mathrm{a}$ \\
\hline C. mollis (Kunth) Spreng. & 0 & 0 & 0 & 0 & 1 & 0 & 0 & a \\
\hline C. platyloba $\mathrm{S}$. Watson & 0 & 0 & 1 & 0 & 0 & 0 & 1 & a \\
\hline C. vesicaria $\mathrm{L}$. & 0 & 0 & 0 & 1 & 0 & 1 & 0 & a \\
\hline C. yucatanensis Greenm. & 0 & 0 & 0 & 1 & 0 & 0 & 0 & a \\
\hline Chamaecrista chamaecristoides (Collad) Greene & 0 & 0 & 1 & 0 & 0 & 0 & 0 & $\mathrm{~h}$ \\
\hline C. diphylla (L.) Greene & 0 & 1 & 0 & 0 & 0 & 0 & 0 & $\mathrm{~h}$ \\
\hline C. flexuosa (L.) Greene & 0 & 1 & 0 & 0 & 0 & 0 & 0 & $\mathrm{~h}$ \\
\hline C. glandulosa (L.) Greene & 0 & 0 & 1 & 0 & 0 & 0 & 0 & ar \\
\hline C. nictitans (L.) Moench & 1 & 0 & 0 & 0 & 0 & 0 & 0 & $\mathrm{~h}$ \\
\hline Cynometra retusa Britton et Rose & 0 & 0 & 0 & 1 & 0 & 0 & 0 & a \\
\hline Dialium guianense (Aubl.) Sandwith & 0 & 0 & 0 & 1 & 1 & 0 & 0 & a \\
\hline Haematoxylum campechianum $\mathrm{L}$. & 0 & 0 & 0 & 1 & 0 & 1 & 0 & $\mathrm{a}$ \\
\hline Hymenaea courbaril L. & 0 & 1 & 0 & 0 & 0 & 0 & 0 & a \\
\hline Senna atomaria (L.) H.S. Irwin et Barneby & 0 & 1 & 0 & 0 & 0 & 0 & 0 & $\mathrm{a}$ \\
\hline S. bicapsularis (L.) Roxb. & 1 & 0 & 0 & 0 & 0 & 0 & 0 & $\mathrm{a}$ \\
\hline S. cobanensis (Britton) H.S. Irwin et Barneby & 0 & 0 & 0 & 1 & 1 & 0 & 0 & $\mathrm{~h} / \mathrm{su}$ \\
\hline S. fruticosa (Mill.) H.S. Irwin et Barneby & 0 & 0 & 0 & 1 & 1 & 0 & 0 & $\mathrm{ar} / \mathrm{a}$ \\
\hline S. hayesiana (Britton et Rose) H.S. Irwin et Barneby & 0 & 0 & 0 & 1 & 1 & 0 & 0 & $\mathrm{ar} / \mathrm{a}$ \\
\hline S. hirsuta (L.) H.S Irwin et Barneby & 0 & 1 & 0 & 0 & 0 & 0 & 0 & $\mathrm{~h}$ \\
\hline S. obtusifolia (L.) H.S. Irwin et Barneby & 0 & 1 & 0 & 0 & 0 & 0 & 0 & $\mathrm{~h}$ \\
\hline S. occidentalis (L.) Link & 0 & 1 & 1 & 0 & 0 & 0 & 0 & $\mathrm{~h} / \mathrm{ar}$ \\
\hline S. pallida (Vahl) H.S. Irwin et Barneby & 0 & 1 & 0 & 0 & 0 & 0 & 0 & $\mathrm{a} / \mathrm{ar}$ \\
\hline S. papillosa (Britton et Rose) H.S. Irwin et Barneby & 0 & 0 & 0 & 1 & 1 & 0 & 0 & ar \\
\hline S. pendula (Willd.) H.S. Irwin et Barneby & 0 & 1 & 0 & 0 & 0 & 0 & 0 & $\mathrm{a} / \mathrm{ar}$ \\
\hline S. pentagonia (Mill.) H.S. Irwin et Barneby & 0 & 0 & 0 & 1 & 1 & 0 & 0 & $\mathrm{H}$ \\
\hline S. peralteana (Kunth) H.S. Irwin et Barneby & 0 & 0 & 0 & 1 & 0 & 0 & 0 & $\mathrm{a} / \mathrm{ar}$ \\
\hline S. polyphylla (Jacq.) H.S. Irwin et Barneby & 0 & 0 & 1 & 0 & 1 & 1 & 0 & ar \\
\hline S. quinquangulata (Rich.) H.S. Irwin et Barneby & 0 & 0 & 0 & 1 & 1 & 0 & 0 & ar \\
\hline S. racemosa (Mill.) H.S. Irwin et Barneby & 0 & 1 & 0 & 0 & 0 & 0 & 0 & $\mathrm{a} / \mathrm{ar}$ \\
\hline S. reticulata (Willd.) H.S. Irwin et Barneby & 0 & 0 & 0 & 1 & 1 & 0 & 0 & ar \\
\hline S. undulata (Benth.) H.S. Irwin et Barneby & 0 & 0 & 0 & 1 & 1 & 0 & 0 & ar \\
\hline S. uniflora (Mill.) H.S. Irwin et Barneby & 0 & 1 & 0 & 0 & 0 & 0 & 0 & $\mathrm{H}$ \\
\hline $\begin{array}{l}\text { S. villosa (Mill.) H.S. Irwin et Barneby } \\
\text { MIMOSACEAE }\end{array}$ & 0 & 0 & 1 & 1 & 0 & 0 & 0 & $\mathrm{~h} / \mathrm{ar}$ \\
\hline Acacia californica Brandegee & 0 & 0 & 1 & 0 & 0 & 0 & 0 & A \\
\hline A. cedilloi L. Rico & 0 & 0 & 0 & 0 & 0 & 0 & 1 & A \\
\hline A. centralis Britton et Rose) Lundell & 0 & 0 & 0 & 0 & 1 & 0 & 0 & A \\
\hline A. chiapensis Saff. & 0 & 0 & 0 & 1 & 0 & 0 & 0 & A \\
\hline A. collinsii Saff. & 0 & 0 & 0 & 1 & 1 & 0 & 0 & A \\
\hline
\end{tabular}


Apéndice. Continúa.

\begin{tabular}{|c|c|c|c|c|c|c|c|c|}
\hline Especie & PAN & $N E O$ & NA & $C A$ & $S A$ & $A N T$ & GUA y $B E L$ & Hábito \\
\hline A. cornigera (L.) Willd. & 0 & 0 & 0 & 1 & 0 & 1 & 0 & A \\
\hline A. dolichostachya S.F. Blake & 0 & 0 & 0 & 0 & 0 & 0 & 1 & A \\
\hline A. farnesiana (L.) Willd. & 0 & 1 & 0 & 0 & 0 & 0 & 0 & A \\
\hline A. gaumeri S.F. Blake & 0 & 0 & 0 & 0 & 0 & 0 & 1 & A \\
\hline A. gentlei Standl. & 0 & 0 & 0 & 1 & 0 & 0 & 0 & A \\
\hline A. globulifera Saff. & 0 & 0 & 0 & 1 & 0 & 0 & 0 & A \\
\hline A. hayesii Benth. & 0 & 0 & 0 & 1 & 1 & 0 & 0 & A \\
\hline A. macrantha Humb. et Bonpl. ex Willd. & 0 & 0 & 1 & 0 & 0 & 0 & 0 & A \\
\hline A. pennatula (Schltdl. et Cham.) Benth. & 0 & 0 & 0 & 1 & 1 & 0 & 0 & A \\
\hline A. polyphylla DC. & 0 & 0 & 0 & 1 & 1 & 0 & 0 & A \\
\hline A. riparia Kunth & 0 & 1 & 0 & 0 & 0 & 0 & 0 & A \\
\hline A. usumacintensis Lundell & 0 & 0 & 0 & 0 & 1 & 0 & 0 & A \\
\hline Acaciella angustissima (Mill.) Britton et Rose & 0 & 1 & 0 & 0 & 0 & 0 & 0 & A \\
\hline A. villosa (Sw.) Britton et Rose & 0 & 1 & 0 & 0 & 0 & 0 & 0 & A \\
\hline Albizia guachapele (Kunth) Dugand & 0 & 0 & 0 & 0 & 1 & 0 & 0 & A \\
\hline A. niopoides (Spruce ex Benth.) Burkart & 0 & 0 & 0 & 1 & 1 & 0 & 0 & A \\
\hline A. tomentosa (Micheli) Standl. & 0 & 0 & 0 & 1 & 0 & 0 & 0 & A \\
\hline Calliandra belizensis (Britton et Rose) Standl. & 0 & 0 & 0 & 0 & 0 & 0 & 1 & A \\
\hline C. caeciliae Harms. & 0 & 0 & 0 & 1 & 0 & 0 & 0 & A \\
\hline C. calothyrsus Meisn. & 0 & 0 & 0 & 1 & 1 & 0 & 0 & A \\
\hline C. houstoniana (Mill.) Standl. & 0 & 0 & 0 & 1 & 0 & 0 & 0 & A \\
\hline C. tergemina (L.) Benth. & 0 & 1 & 0 & 0 & 0 & 0 & 0 & A \\
\hline Chloroleucon mangense (Jacq.) Britton et Rose & 0 & 0 & 1 & 0 & 0 & 0 & 0 & A \\
\hline Cojoba arborea $(\mathrm{L}$.$) Britton et Rose$ & 0 & 0 & 1 & 0 & 0 & 0 & 0 & A \\
\hline C. graciliflora (S.F. Blake) Britton et Rose & 0 & 0 & 0 & 1 & 0 & 0 & 0 & A \\
\hline Desmanthus leptophyllus Kunth & 0 & 1 & 0 & 0 & 0 & 0 & 0 & $\mathrm{~h} / \mathrm{su}$ \\
\hline D. paspalaceus (Lindm.) Burkart & 0 & 0 & 0 & 0 & 1 & 0 & 0 & $\mathrm{~h} / \mathrm{su}$ \\
\hline D. pubescens B.L. Turner & 0 & 0 & 0 & 1 & 0 & 1 & 0 & $\mathrm{~h} / \mathrm{su}$ \\
\hline D. virgatus (L.) Willd. & 0 & 1 & 0 & 0 & 0 & 0 & 0 & $\mathrm{H}$ \\
\hline Ebenopsis ebano (Berland.) Barneby et J.W. Grimes & 0 & 0 & 1 & 0 & 0 & 0 & 0 & A \\
\hline Enterolobium cyclocarpum (Jacq.) Griseb. & 0 & 0 & 0 & 1 & 1 & 0 & 0 & A \\
\hline Havardia albicans (Kunth) Britton et Rose & 0 & 0 & 0 & 0 & 0 & 0 & 1 & A \\
\hline H. pallens (Benth.) Britton et Rose & 0 & 0 & 1 & 0 & 0 & 0 & 0 & A \\
\hline Havardia platyloba (Bertero ex DC.) Britton et Rose & 0 & 0 & 0 & 1 & 1 & 0 & 0 & A \\
\hline Inga jinicuil Schltdl. et Cham. ex G. Don & 0 & 0 & 0 & 1 & 1 & 0 & 0 & A \\
\hline I. punctata Willd. & 0 & 0 & 0 & 0 & 1 & 0 & 0 & A \\
\hline I. vera Willd. & 0 & 1 & 0 & 0 & 0 & 0 & 0 & A \\
\hline Leucaena leucocephala (Lam.) de Wit & 0 & 0 & 1 & 0 & 0 & 0 & 0 & A \\
\hline L. shannonii Donn. Sm. & 0 & 0 & 0 & 1 & 0 & 0 & 0 & A \\
\hline Lysiloma acapulcense (Kunth) Benth. & 0 & 0 & 1 & 1 & 0 & 0 & 0 & A \\
\hline L. latisiliquum (L.) Benth. & 0 & 0 & 1 & 0 & 1 & 1 & 0 & A \\
\hline Mimosa affinis B.L. Rob. & 0 & 1 & 0 & 0 & 0 & 0 & 0 & $\mathrm{~h} / \mathrm{su}$ \\
\hline M. albida Humb. et Bonpl. ex Willd. & 0 & 0 & 0 & 1 & 1 & 0 & 0 & $\mathrm{a} /$ th \\
\hline M. bahamensis Benth. & 0 & 0 & 0 & 1 & 0 & 1 & 0 & $\mathrm{a}$ \\
\hline M. distachya Cav. & 0 & 1 & 0 & 0 & 0 & 0 & 0 & ar \\
\hline M. guatemalensis (Hook. et Arn.) Benth. & 0 & 0 & 0 & 1 & 0 & 0 & 0 & $\mathrm{~h} / \mathrm{ar}$ \\
\hline M. orthocarpa Spruce ex Benth. & 0 & 0 & 0 & 0 & 1 & 0 & 0 & ar \\
\hline M. pigra $\mathrm{L}$. & 1 & 0 & 0 & 0 & 0 & 0 & 0 & ar \\
\hline M. pudica $\mathrm{L}$. & 0 & 0 & 0 & 0 & 1 & 0 & 0 & $\mathrm{~h} / \mathrm{su}$ \\
\hline M. somnians Humb. et Bonpl. ex Willd. & 0 & 0 & 0 & 1 & 1 & 0 & 0 & $\mathrm{~h} / \mathrm{su}$ \\
\hline Neptunia natans (L. f.) Druce & 1 & 0 & 0 & 0 & 0 & 0 & 0 & $\mathrm{~h}$ \\
\hline
\end{tabular}


Apéndice. Continúa.

\begin{tabular}{|c|c|c|c|c|c|c|c|c|}
\hline Especie & $P A N$ & $N E O$ & $N A$ & $C A$ & $S A$ & $A N T$ & $G U A$ y $B E L$ & Hábito \\
\hline N. plena (L.) Benth. & 1 & 0 & 0 & 0 & 0 & 0 & 0 & $\mathrm{~h}$ \\
\hline Piptadenia flava (Spreng. ex DC.) Benth. & 0 & 0 & 0 & 1 & 1 & 0 & 0 & ar \\
\hline P. viridiflora (Kunth) Benth. & 0 & 0 & 0 & 0 & 1 & 0 & 0 & $\mathrm{a}$ \\
\hline Pithecellobium dulce (Roxb.) Benth. & 0 & 1 & 1 & 0 & 0 & 0 & 0 & a \\
\hline P. keyense Britton & 0 & 0 & 1 & 0 & 0 & 1 & 0 & $\mathrm{a}$ \\
\hline P. lanceolatum Benth. & 0 & 0 & 0 & 1 & 1 & 0 & 0 & $\mathrm{a}$ \\
\hline P. seleri Harms. & 0 & 0 & 0 & 1 & 0 & 0 & 0 & $\mathrm{a}$ \\
\hline P. unguis-cati (L.) Benth. & 0 & 1 & 0 & 0 & 0 & 0 & 0 & $\mathrm{a}$ \\
\hline P. winzerlingii Britton et Rose & 0 & 0 & 0 & 1 & 0 & 0 & 0 & $\mathrm{a}$ \\
\hline Prosopis juliflora (Sw.) DC. & 0 & 0 & 1 & 0 & 0 & 0 & 0 & $\mathrm{a}$ \\
\hline Zapoteca formosa (Kunth) H.M. Hern. & 0 & 1 & 0 & 0 & 0 & 0 & 0 & ar \\
\hline Zygia recordii Britton et Rose & 0 & 0 & 0 & 1 & 0 & 0 & 0 & $\mathrm{a}$ \\
\hline $\begin{array}{l}\text { Z. stevensonii (Standl.) Killip ex Record } \\
\text { PAPILIONOIDEAE }\end{array}$ & 0 & 0 & 0 & 0 & 0 & 0 & 1 & $\mathrm{a}$ \\
\hline Abrus precatorius L. & 1 & 0 & 0 & 0 & 0 & 0 & 0 & th \\
\hline Acosmium panamense (Benth.) Yakoviev & 0 & 0 & 0 & 1 & 1 & 0 & 0 & $\mathrm{a}$ \\
\hline Aeschynomene americana $\mathrm{L}$. & 0 & 1 & 0 & 0 & 0 & 0 & 0 & ar \\
\hline A. fascicularis Schltdl. et Cham. & 0 & 0 & 0 & 1 & 1 & 0 & 0 & $\mathrm{~h}$ \\
\hline A. rudis Benth. & 0 & 1 & 0 & 1 & 1 & 0 & 0 & $\mathrm{~h} / \mathrm{su}$ \\
\hline A. sensitiva $\mathrm{Sw}$ & 1 & 0 & 0 & 0 & 0 & 0 & 0 & $\mathrm{~h}$ \\
\hline Andira inermis (W. Wright) Kunth ex DC. & 1 & 0 & 0 & 0 & 0 & 0 & 0 & $\mathrm{a}$ \\
\hline Apoplanesia paniculata C. Presl & 0 & 0 & 0 & 1 & 1 & 0 & 0 & $\mathrm{a}$ \\
\hline Ateleia cubensis Griseb. & 0 & 0 & 0 & 0 & 0 & 1 & 1 & a \\
\hline A. pterocarpa Moc. et Sessé ex D. Dietr. & 0 & 0 & 0 & 1 & 0 & 0 & 0 & a \\
\hline Cajanus cajan (L.) Millsp. & 1 & 0 & 0 & 0 & 0 & 0 & 0 & $\mathrm{tl}$ \\
\hline Canavalia brasiliensis Mart. ex Benth. & 0 & 1 & 0 & 0 & 0 & 0 & 0 & th \\
\hline C. rosea (Sw.) DC. & 1 & 0 & 0 & 0 & 0 & 0 & 0 & th \\
\hline C. villosa Benth. & 0 & 0 & 0 & 1 & 1 & 0 & 0 & $\mathrm{tl}$ \\
\hline Calopogonium mucunoides Desv. & 0 & 1 & 0 & 0 & 0 & 0 & 0 & $\mathrm{tl}$ \\
\hline Centrosema angustifolium (Kunth) Benth. & 0 & 0 & 0 & 1 & 1 & 0 & 0 & $\mathrm{tl}$ \\
\hline C. macrocarpum Benth. & 0 & 1 & 0 & 0 & 0 & 0 & 0 & $\mathrm{tl}$ \\
\hline C. molle Mart. ex Benth. & 0 & 1 & 0 & 0 & 0 & 0 & 0 & $\mathrm{tl}$ \\
\hline C. pascuorum Mart. ex Benth. & 0 & 0 & 0 & 1 & 1 & 0 & 0 & $\mathrm{tl}$ \\
\hline C. plumieri (Turpin ex Pers.)Benth. & 0 & 1 & 0 & 0 & 0 & 0 & 0 & $\mathrm{tl}$ \\
\hline C. sagittatum (Willd.) L. Riley & 0 & 1 & 0 & 0 & 0 & 0 & 0 & $\mathrm{tl}$ \\
\hline C. schottii (Millsp.) K. Schum. & 0 & 0 & 0 & 1 & 1 & 0 & 0 & $\mathrm{tl}$ \\
\hline C. unifoliatum (Rose) Lundell & 0 & 0 & 0 & 1 & 0 & 0 & 0 & $\mathrm{tl}$ \\
\hline C. virginianum (L.) Benth. & 0 & 1 & 1 & 0 & 0 & 0 & 0 & $\mathrm{tl}$ \\
\hline Chaetocalyx scandens (L.) Urb. & 0 & 1 & 0 & 0 & 0 & 0 & 0 & $\mathrm{tl}$ \\
\hline Clitoria falcata Lam. & 0 & 1 & 0 & 0 & 0 & 0 & 0 & th \\
\hline Coursetia caribaea (Jacq.) Lavin & 0 & 1 & 0 & 0 & 0 & 0 & 0 & $\mathrm{~h} / \mathrm{su}$ \\
\hline Crotalaria cajanifolia Kunth & 0 & 1 & 0 & 0 & 0 & 0 & 0 & ar \\
\hline C. incana $\mathrm{L}$. & 0 & 1 & 1 & 0 & 0 & 0 & 0 & ar \\
\hline C. longirostrata Hook. et Arn. & 0 & 0 & 0 & 1 & 0 & 0 & 0 & ar \\
\hline C. pumila Ortega & 0 & 1 & 0 & 0 & 0 & 0 & 0 & ar \\
\hline C. purdiana H. Senn & 0 & 0 & 0 & 0 & 1 & 1 & 0 & $\mathrm{~h}$ \\
\hline C. retusa $\mathrm{L}$. & 1 & 0 & 0 & 0 & 0 & 0 & 0 & $\mathrm{~h}$ \\
\hline C. verrucosa $\mathrm{L}$. & 1 & 0 & 0 & 0 & 0 & 0 & 0 & ar \\
\hline Dalbergia brownei (Jacq.) Schinz & 0 & 0 & 1 & 0 & 0 & 0 & 0 & $\mathrm{a} / \mathrm{ar}$ \\
\hline D. ecastaphyllum (L.) Taub. & 0 & 1 & 1 & 0 & 0 & 0 & 0 & $\mathrm{a} / \mathrm{ar}$ \\
\hline D. glabra (Mill.) Standl. & 0 & 0 & 0 & 1 & 0 & 0 & 0 & $\mathrm{a} / \mathrm{ar}$ \\
\hline
\end{tabular}


Apéndice. Continúa.

\begin{tabular}{|c|c|c|c|c|c|c|c|c|}
\hline Especie & $P A N$ & NEO & $N A$ & $C A$ & $S A$ & $A N T$ & $G U A$ y $B E L$ & Hábito \\
\hline Dalea carthagenensis (Jacq.) J.F. Macbr. & 0 & 1 & 0 & 0 & 0 & 0 & 0 & ar \\
\hline D. scandens (Mill.) R.T. Clausen & 0 & 0 & 1 & 0 & 0 & 1 & 0 & ar \\
\hline Desmodium affine Schltdl. & 0 & 1 & 0 & 0 & 0 & 0 & 0 & $\mathrm{~h}$ \\
\hline D. distortum (Aubl.) J.F. Macbr. & 0 & 0 & 0 & 1 & 1 & 0 & 0 & $\mathrm{~h}$ \\
\hline D. glabrum (Mill.) DC. & 0 & 1 & 0 & 0 & 0 & 0 & 0 & $\mathrm{~h}$ \\
\hline D. incanum DC. & 0 & 1 & 0 & 0 & 0 & 0 & 0 & $\mathrm{~h} / \mathrm{su}$ \\
\hline D. procumbens (Mill.) Hitchc. & 0 & 1 & 0 & 0 & 0 & 0 & 0 & $\mathrm{~h}$ \\
\hline D. scorpiurus (Sw.) Desv. & 0 & 1 & 0 & 0 & 0 & 0 & 0 & $\mathrm{~h}$ \\
\hline D. tortuosum (Sw.) DC. & 0 & 1 & 1 & 0 & 0 & 0 & 0 & $\mathrm{~h}$ \\
\hline D. triflorum (L.) DC. & 1 & 0 & 0 & 0 & 0 & 0 & 0 & $\mathrm{~h}$ \\
\hline Dioclea wilsonii Standl. & 0 & 0 & 0 & 1 & 1 & 0 & 0 & tl \\
\hline Diphysa carthagenensis Jacq. & 0 & 0 & 0 & 1 & 1 & 0 & 0 & $\mathrm{a}$ \\
\hline D. paucifoliolata R. Antonio et M. Sousa & 0 & 0 & 0 & 0 & 0 & 0 & 1 & ar \\
\hline D. yucatanensis A. Hanan et M. Sousa & 0 & 0 & 0 & 0 & 0 & 0 & 1 & ar \\
\hline Erythrina standleyana Krukoff & 0 & 0 & 0 & 1 & 0 & 1 & 0 & $\mathrm{a} / \mathrm{ar}$ \\
\hline Galactia jussiaeana Kunth & 0 & 1 & 0 & 0 & 0 & 0 & 0 & $\mathrm{su}$ \\
\hline G. multiflora B.L. Rob. & 0 & 0 & 1 & 1 & 0 & 0 & 0 & th \\
\hline G. spiciformis Torr. et A. Gray & 0 & 0 & 1 & 0 & 0 & 1 & 0 & th \\
\hline G. striata (Jacq.) Urb. & 0 & 1 & 1 & 0 & 0 & 0 & 0 & th \\
\hline Gliricidia sepium (Jacq.) Kunth ex Walp. & 0 & 1 & 0 & 0 & 0 & 0 & 0 & $\mathrm{a}$ \\
\hline Harpalyce arborescens A. Gray & 0 & 1 & 0 & 0 & 0 & 0 & 0 & $\mathrm{ar} / \mathrm{a}$ \\
\hline Indigofera jamaicensis Spreng. & 0 & 1 & 0 & 0 & 0 & 0 & 0 & $\mathrm{ar} / \mathrm{h}$ \\
\hline I. lespedezioides Kunth & 0 & 1 & 0 & 0 & 0 & 0 & 0 & $\mathrm{ar} / \mathrm{h}$ \\
\hline I. suffruticosa Mill. & 0 & 1 & 1 & 0 & 0 & 0 & 0 & ar \\
\hline I. thibaudiana DC. & 0 & 0 & 0 & 1 & 0 & 0 & 0 & ar \\
\hline I. tinctoria $\mathrm{L}$. & 0 & 1 & 1 & 0 & 0 & 0 & 0 & $\mathrm{~h}$ \\
\hline Lennea melanocarpa (Schltdl.) Vatke ex Harms. & 0 & 0 & 0 & 1 & 0 & 0 & 0 & $\mathrm{a} / \mathrm{ar}$ \\
\hline Lonchocarpus castilloi Standl. & 0 & 0 & 0 & 1 & 0 & 0 & 0 & $\mathrm{a}$ \\
\hline L. guatemalensis Benth. & 0 & 0 & 1 & 1 & 0 & 0 & 0 & a \\
\hline L. hondurensis Benth. & 0 & 0 & 0 & 1 & 0 & 0 & 0 & a \\
\hline L. luteomaculatus Pittier & 0 & 0 & 0 & 1 & 0 & 0 & 0 & a \\
\hline L. punctatus Kunth & 0 & 1 & 0 & 0 & 0 & 0 & 0 & $\mathrm{a}$ \\
\hline L. rugosus Benth. & 0 & 1 & 0 & 1 & 0 & 0 & 0 & a \\
\hline L. $x \boldsymbol{u} u \boldsymbol{l}$ Lundell & 0 & 0 & 0 & 0 & 0 & 0 & 1 & $\mathrm{a}$ \\
\hline L. yucatanensis Pittier & 0 & 0 & 0 & 0 & 0 & 0 & 1 & $\mathrm{a}$ \\
\hline Machaerium biovulatum Micheli & 0 & 0 & 0 & 1 & 1 & 0 & 0 & $\mathrm{a} / \mathrm{ar} / \mathrm{tl}$ \\
\hline M. cirrhiferum Pittier & 0 & 0 & 0 & 1 & 0 & 0 & 0 & $\mathrm{a} / \mathrm{ar}$ \\
\hline M. falciforme Rudd & 0 & 0 & 0 & 1 & 0 & 0 & 0 & $\mathrm{a} / \mathrm{ar}$ \\
\hline M. isadelphum (E. Mey) Amshoff & 0 & 0 & 0 & 1 & 1 & 0 & 0 & $\mathrm{ar} / \mathrm{ar} / \mathrm{tl}$ \\
\hline M. kegelii Meisn. & 0 & 0 & 0 & 1 & 1 & 0 & 0 & $\mathrm{a} / \mathrm{ar} / \mathrm{tl}$ \\
\hline M. riparium Brandegee & 0 & 0 & 0 & 1 & 0 & 0 & 0 & $\mathrm{tl}$ \\
\hline M. seemannii Benth. & 0 & 0 & 0 & 1 & 1 & 0 & 0 & $\mathrm{a} / \mathrm{a} / \mathrm{tl}$ \\
\hline Macroptiulium atropurpureum (Sessé et Moc ex DC.) Urb. & 0 & 1 & 1 & 0 & 0 & 0 & 0 & $\mathrm{~h} / \mathrm{th}$ \\
\hline M. gracile (Poepp. ex Benth.) Urb. & 0 & 0 & 1 & 0 & 0 & 0 & 0 & $\mathrm{~h}$ \\
\hline M. lathyroides (L.) Urb. & 0 & 1 & 0 & 0 & 0 & 0 & 0 & $\mathrm{~h}$ \\
\hline M. longepedunculatum (Mart. ex Benth.) Urb. & 0 & 1 & 0 & 0 & 0 & 0 & 0 & $\mathrm{~h} / \mathrm{th}$ \\
\hline Marina scopa Barneby & 0 & 1 & 0 & 0 & 0 & 0 & 0 & ar \\
\hline Mucuna holtonii (Kuntze) Moldenke & 0 & 0 & 0 & 1 & 1 & 0 & 0 & $\mathrm{tl}$ \\
\hline Myroxylon balsamum (L.) Harms & 0 & 0 & 0 & 1 & 1 & 0 & 0 & ar \\
\hline Nissolia fruticosa Jacq. & 0 & 1 & 0 & 0 & 0 & 0 & 0 & $\mathrm{th} / \mathrm{tl}$ \\
\hline Oxyrhynchus volubilis Brandegee & 0 & 0 & 1 & 0 & 0 & 1 & 0 & $\mathrm{tl}$ \\
\hline
\end{tabular}


Apéndice. Continúa.

\begin{tabular}{|c|c|c|c|c|c|c|c|c|}
\hline Especie & $P A N$ & $N E O$ & $N A$ & $C A$ & $S A$ & $A N T$ & $G U A$ y $B E L$ & Hábito \\
\hline Pachyrhizus erosus (L.) Urb. & 0 & 1 & 0 & 0 & 0 & 0 & 0 & $\mathrm{tl}$ \\
\hline Piscidia piscipula (L.) Sarg. & 0 & 0 & 1 & 1 & 0 & 1 & 0 & $\mathrm{a}$ \\
\hline Platymiscium yucatanum Standl. & 0 & 0 & 0 & 0 & 0 & 0 & 1 & $\mathrm{a}$ \\
\hline Pterocarpus rohrii Vahl & 0 & 0 & 0 & 1 & 1 & 0 & 0 & th \\
\hline Rhynchosia americana (Mill.) Metz & 0 & 0 & 0 & 1 & 0 & 0 & 0 & th \\
\hline R. longiracemosa M. Martens et Galeotti & 0 & 0 & 0 & 1 & 0 & 0 & 0 & th \\
\hline R. minima (L.) DC. & 0 & 1 & 0 & 0 & 0 & 0 & 0 & th \\
\hline R. reticulata (Sw.) DC. & 0 & 1 & 0 & 0 & 0 & 0 & 0 & th \\
\hline R. swartzii (Vail) Urb. & 0 & 0 & 1 & 1 & 0 & 1 & 0 & th \\
\hline R. yucatanensis Grear & 0 & 0 & 0 & 0 & 0 & 0 & 0 & th \\
\hline Sesbania herbacea (Mill.) McVaugh & 0 & 1 & 1 & 0 & 0 & 0 & 0 & ar \\
\hline Sophora tomentosa $\mathrm{L}$. & 0 & 1 & 0 & 0 & 0 & 0 & 0 & $\mathrm{~h}$ \\
\hline Stylosanthes calcicola Small & 0 & 0 & 1 & 1 & 0 & 1 & 0 & $\mathrm{~h}$ \\
\hline S. hamata (L.) Taub. & 0 & 1 & 1 & 0 & 0 & 0 & 0 & $\mathrm{~h}$ \\
\hline S. humilis Kunth & 0 & 1 & 0 & 0 & 0 & 0 & 0 & $\mathrm{~h} / \mathrm{su}$ \\
\hline S. yucatanenses Gama et Dávila & 0 & 0 & 0 & 0 & 0 & 0 & 1 & ar \\
\hline Swartia cubensis (Britton et P. Wilson) Standl. & 0 & 0 & 0 & 1 & 0 & 1 & 0 & $\mathrm{a}$ \\
\hline Tephrosia cinerea (L.) Pers. & 0 & 1 & 0 & 0 & 0 & 0 & 0 & $\mathrm{~h}$ \\
\hline Teramnus uncinatus (L.) Sw. & 0 & 1 & 0 & 0 & 0 & 0 & 0 & th \\
\hline Vatairea lundellii (Standl.) Killip ex Record & 0 & 0 & 0 & 1 & 0 & 0 & 0 & $\mathrm{a}$ \\
\hline Vigna adenantha (G. Mey.) Maréchal, Mascherpa et Stainier & 0 & 1 & 0 & 0 & 0 & 0 & 0 & $\mathrm{th} / \mathrm{tl}$ \\
\hline Vigna candida (Vell) Maréchal, Mascherpa et Stainier & 0 & 0 & 0 & 1 & 1 & 0 & 0 & th \\
\hline V. elegans (Piper) Maréchal, Mascherpa et Stainier & 0 & 0 & 0 & 1 & 0 & 1 & 0 & th \\
\hline V. luteola (Jacq.) Benth. & 0 & 1 & 0 & 0 & 0 & 0 & 0 & $\mathrm{th} / \mathrm{tl}$ \\
\hline V. unguiculata (L.) Walp. & 0 & 1 & 1 & 0 & 0 & 0 & 0 & th/su \\
\hline V. vexillata (L.) A. Rich. & 0 & 1 & 0 & 0 & 0 & 0 & 0 & th \\
\hline Zornia gemella Vogel & 0 & 1 & 1 & 0 & 0 & 0 & 0 & $\mathrm{~h}$ \\
\hline TOTALES & 14 & 76 & $43 / 11$ & $90 / 32$ & $55 / 8$ & $21 / 1$ & $15 / 15$ & \\
\hline
\end{tabular}

